\title{
Natural convection in a horizontal cylinder with axial rotation
}

\author{
Odalys Sánchez, ${ }^{*}$ Isabel Mercader, ${ }^{\dagger}$ Oriol Batiste, ${ }^{\ddagger}$ and Arantxa Alonso ${ }^{\S}$ \\ Departament de Física, Universitat Politècnica de Catalunya, Mòdul B4, 08034 Barcelona, Spain
}

(Received 14 March 2016; published 23 June 2016)

\begin{abstract}
We study the problem of thermal convection in a laterally heated horizontal cylinder rotating about its axis. A cylinder of aspect ratio $\Gamma=H / 2 R=2$ containing a small Prandtl number fluid $(\sigma=0.01)$ representative of molten metals and molten semiconductors at high temperature is considered. We focus on a slow rotation regime $(\Omega<8)$, where the effects of rotation and buoyancy forces are comparable. The Navier-Stokes and energy equations with the Boussinesq approximation are solved numerically to calculate the basic states, analyze their linear stability, and compute several secondary flows originated from the instabilities. Due to the confined cylindrical geometry - the presence of lateral walls and lids-all the flows are completely three dimensional, even the basic steady states. Results characterizing the basic states as the rotation rate increases are presented. As it occurred in the nonrotating case for higher values of the Prandtl number, two curves of steady states with the same symmetric character coexist for moderate values of the Rayleigh number. In the range of $\Omega$ considered, rotation has a stabilizing effect only for very small values. As the value of the rotation rate approaches $\Omega=3.5$ and $\Omega=4.5$, the scenario of bifurcations becomes more complex due to the existence in both cases of very close bifurcations of codimension 2 , which in the latter case involve both curves of symmetric solutions.
\end{abstract}

DOI: 10.1103/PhysRevE.93.063113

\section{INTRODUCTION}

The problem of convection induced by a lateral temperature gradient has been extensively studied during the past few decades. The main interest in studying this system comes from heat transfer problems of industrial interest and from the study of the melt zone in crystal growth processes, like the horizontal Bridgman method. In this latter problem, crystals grown from the melt can present inhomogeneities in the form of striations, caused by oscillatory variations of the concentration in the solidification front [1]. For this reason, many studies have focused on the study of the oscillatory threshold in low-Prandtl-number fluids in different geometrical configurations [2-7]. The system is also interesting from a fundamental fluid dynamics point of view, since it exhibits a rich nonlinear behavior that leads to complex spatiotemporal dynamics [8-11]. A bounded cylinder can be used to represent realistically the melt zone in the horizontal Bridgman crystal growth process. Such a system was studied by Vaux et al. [3] for a fixed value of the Prandtl number, $\sigma=0.026$, and different cell sizes, and was recently analyzed in the work of Mercader et al. [12] for a cylinder of aspect ratio $\Gamma=H / 2 R=2$ and Prandtl numbers in the range $\sigma<0.026$.

However, the superposition of rotation along the cylinder axis has received less attention, despite providing a possible mechanism for the stabilization and homogenization of the flow, which could be relevant in different crystal growth techniques involving horizontal cylindrical geometries. In addition, including rotation increases the interest of the configuration, due to the two competing mechanisms at stake that may result in a richer dynamical phenomenology.

The numerical works addressing the study of convection in a rotating horizontal cylinder are scarce. The pioneering work

\footnotetext{
*odalys.sanchez@upc.edu

${ }^{\dagger}$ maria.isabel.mercader@upc.edu

‡oriol@fa.upc.edu

§arantxa@fa.upc.edu
}

of Yang et al. [13], aimed at studying crystal growth processes using chemical vapour deposition, establishes clearly the effect of rotation on the basic flow for a horizontal cylinder of aspect ratio 2, with air as working fluid. At low rotational speeds, they observe that rotation tilts the temperature field in the lateral planes as a result of the Coriolis force. With increasing rotation, temperature becomes more uniform in space and the strength of the flow due to buoyancy in the vertical plane reduces. At sufficiently high rotation rates, the fluid acts as a solid body. Fujiwara et al. [14] consider the same system for two-component fluids of Prandtl numbers 0.73 and 0 . They identify a transition from a boundary layer driven flow to a core driven flow as the rotation rate increases and the fluid tends to behave as a solid body. Lang and Tu [15] control the radial and axial dopant segregation by slow rotation in microgravity Bridgman growth of gallium-doped germanium at two different gravity orientations. They investigate the feasibility of using slow rotation to suppress the g-jitter effect. They found an optimum rotation speed that gives rise to a uniform dopant distribution.

Other works devoted to this problem are motivated by the thermal processing of canned liquids in the food industry. Foluso and Torrance [16] present a detailed study of a cylinder subjected to radial and normal gravity fields with uniform volumetric heating and an isothermal outer boundary. When the effects of rotation and buoyancy are comparable, they observe complex time-dependent flows, but when rotation dominates, the flow assumes a solid body rotation and the temperature field approaches that of pure conduction. Bearing the same motivation of food industry in mind, the work of Zhao et al. [17] uses a simpler approach, since the flow in the horizontal cylinder is assumed to be two-dimensional in vertical planes. Results of the numerical computations are compared with a perturbative analysis and confirm the existence of the shear regime, with a Nusselt number much larger than unity, and the solid body rotation regime, with a convective heat transfer considerably reduced to almost its value in the pure conduction regime. 
Our present work incorporates rotation to the system analyzed in Ref. [12] and focuses on a range of rotation rates where natural convection is not yet dominated by rotational effects. Our aim is to check to what extent rotation can be used as a new method in crystal growth processes. On one hand, rotation is expected to delay the onset of oscillatory instabilities, and this might be useful to minimize the inhomogeneities in the solidification front associated to them. On the other, it might reduce the longitudinal convective transport, which would result in an homogenization of the heat transfer rate over the solidification front (end wall surface). Specifically, we will consider a differentially heated horizontal cylinder of aspect ratio 2, rotating along its axis and containing a low-Prandtl-number fluid $(\sigma=0.01)$, which is representative of molten metals at high temperature.

The system is described by the Navier-Stokes and energy equations in the laboratory frame under the Boussinesq approximation. Unlike in the nonrotating case, the system only possesses one reflection symmetry, a point symmetry with respect to the center of the cylinder. We will use different numerical tools based on the use of pseudospectral methods to analyze the problem: direct numerical simulation, linear stability analysis, and continuation of steady solution branches with respect to either the Rayleigh number or the rotation rate. We will calculate the basic steady flow patterns, which are invariant with respect to the reflection symmetry of the system, for different rotation rates, and analyze the stability of such flows as the driving thermal gradient is increased. The secondary flows originating from these instabilities can be either stationary or oscillatory and will also be characterized. We will focus on slow rotation regimes, where the kinetic energy of the imposed rotation is comparable to the kinetic energy produced by the buoyancy-induced convection. Detailed bifurcation diagrams will be constructed in a region where different steady states coexist for the same parameter values.

The paper is organized as follows. In Sec. II the problem is stated; we present the model equations in Sec. II A and the numerical methods used in Sec. II B. In Sec. III the main results of the work are presented and discussed; in Sec. III A we describe the symmetries of the equations and analyze the main characteristics of the basic state when the rotation rate increases. In Sec. III B we analyze the stability of the basic flow for rotation rates $\Omega \in(0,4.6)$ and present the corresponding bifurcation diagrams, in Sec. III C we present results of an energy analysis at some critical points, and Sec. IIID is devoted to the computation and description of several saturated secondary flows representative of some of the instabilities found in Sec. III B. Finally, we summarize and outline the relevance of our results in Sec. IV.

\section{THE PHYSICAL PROBLEM}

\section{A. Equations and boundary conditions}

We consider the flow in a laterally heated, finite, horizontal cylinder rotating around the axis. The cylinder of length $H$ and radius $R$ is in the presence of a vertical gravitational force. The $z$ axis is taken along the axis of the cylinder, the origin of coordinates is located on the left lid, and the $x$ axis is parallel

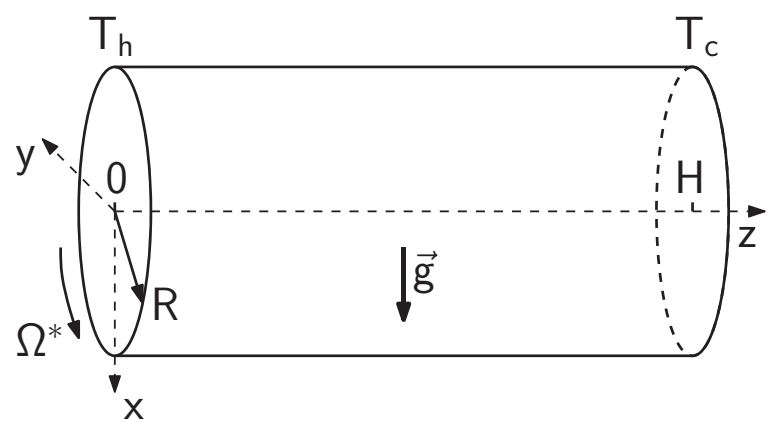

FIG. 1. Configuration of the laterally heated horizontal cylinder and reference frame. Notice that the gravity field is parallel to the $x$ axis.

to the gravitational force, $\mathbf{g}=g \hat{\mathbf{x}}$ (see Fig. 1). The cylinder is heated from the left, being $\Delta T$ the temperature difference between the lids of the cylinder, and rotates around its axis $(z$ axis) at angular velocity $-\Omega^{*} \hat{\mathbf{z}}$. We split the temperature field into two parts, a linear profile and a fluctuation, $\Theta^{*}$,

$$
T=T_{c}+\Delta T(1-z / H)+\Theta^{*},
$$

where $T_{c}$ is temperature at the cold lid $(z=H)$. We consider that the density varies linearly with temperature,

$$
\rho=\rho_{0}\left[1-\alpha\left(T-T_{0}\right)\right],
$$

where $\alpha$ is the thermal expansion coefficient, $T_{0}$ is the mean temperature, and $\rho_{0}$ is the density at temperature $T_{0}$.

Scaling lengths with a reference length $l$, time with the thermal diffusion time $l^{2} / \kappa, \kappa$ being the thermal diffusivity, and temperature with $\Delta T$, the nondimensional Navier-Stokes equations with the Boussinesq approximation written in the laboratory frame that describe the evolution of the velocity field $\mathbf{u}$ and the deviation of the temperature $\Theta=\Theta^{*} / \Delta T$ from the linear profile are as follows:

$$
\begin{gathered}
\nabla \cdot \mathbf{u}=0 \\
\partial_{t} \mathbf{u}+(\mathbf{u} \cdot \nabla) \mathbf{u}=-\nabla p+\sigma \nabla^{2} \mathbf{u}+\operatorname{Ra} \sigma\left(\frac{l}{H} z-\Theta\right) \hat{\mathbf{x}} \\
\partial_{t} \Theta+(\mathbf{u} \cdot \nabla) \Theta=w \frac{l}{H}+\nabla^{2} \Theta .
\end{gathered}
$$

Here $w$ is the $z$ component of the velocity field and $p$ is the nondimensional kinematic pressure including the terms of the gravitational force that can be written as a gradient. The two dimensionless numbers that describe thermal convection are the Rayleigh number Ra and the Prandtl number $\sigma$, defined as

$$
\mathrm{Ra}=\frac{\alpha \Delta T g l^{3}}{\kappa v}, \sigma=\frac{v}{\kappa},
$$

where $v$ is the kinematic viscosity. The Rayleigh number is one of the control parameters of the system and measures the strength of the imposed temperature gradient, and the Prandtl number relates momentum diffusion to thermal diffusion. 
The previous nondimensional equations for the velocity field $\mathbf{u}=(u, v, w)$ in cylindrical coordinates $(r, \theta, z)$ are written as follows:

$$
\nabla \cdot \mathbf{u}=0
$$

$$
\partial_{t} u+[(\mathbf{u} \cdot \nabla) \mathbf{u}]_{r}=-\partial_{r} p+\sigma\left[\nabla^{2} \mathbf{u}\right]_{r}+\operatorname{Ra} \sigma(z / \Gamma-\Theta) \cos \theta,
$$

$$
\partial_{t} v+[(\mathbf{u} \cdot \nabla) \mathbf{u}]_{\theta}=-\frac{1}{r} \partial_{\theta} p+\sigma\left[\nabla^{2} \mathbf{u}\right]_{\theta}-\operatorname{Ra} \sigma(z / \Gamma-\Theta) \sin \theta,
$$

$$
\partial_{t} w+[(\mathbf{u} \cdot \nabla) \mathbf{u}]_{z}=-\partial_{z} p+\sigma\left[\nabla^{2} \mathbf{u}\right]_{z},
$$$$
\partial_{t} \Theta+(\mathbf{u} \cdot \nabla) \Theta=w / \Gamma+\nabla^{2} \Theta,
$$

where $\Gamma=H /(2 R)$ is the aspect ratio of the rotating cylinder. We consider no-slip and fixed temperature boundary conditions at the left and right lids of the horizontal cylinder and no-slip and insulating boundary conditions on the lateral wall. The boundary conditions for the nondimensional fields are written as

$$
\begin{aligned}
& u=w=\Theta=0, v=\Omega r \quad \text { on } \quad z=0, H / l, \\
& u=w=\partial_{r} \Theta=0, v=\Omega R / l \quad \text { on } \quad r=R / l,
\end{aligned}
$$

with $\Omega$ being the nondimensional rotation rate, $\Omega=\Omega^{*} l^{2} / \kappa$. As a measure of the relative heat transported by convection, we will use the Nusselt number $\mathrm{Nu}$ evaluated at the cooler wall of the cavity. This corresponds to computing the ratio of the total heat flux through the right lid to the flux that would correspond to a linear temperature profile. Thus, the Nusselt number has the following expression in nondimensional variables:

$$
\mathrm{Nu}=1-\frac{H /\left.l \int_{0}^{2 \pi} \int_{0}^{R / l} \partial_{z} \Theta\right|_{z=H / l} r d r d \theta}{\pi(R / l)^{2}} .
$$

As a measure of the strength of convection we will use the dimensionless mean kinetic energy defined as

$$
E_{k}=\frac{\int_{0}^{2 \pi} \int_{0}^{R / l} \int_{0}^{H / l} \mathbf{u} \cdot \mathbf{u} r \mathrm{~d} r d \theta d z}{2 \pi(R / l)^{2}(H / l)} .
$$

We choose as reference length the diameter of the cylinder $l=2 R$. In this paper we consider a moderate aspect ratio, $\Gamma=2$, a small Prandtl number fluid, $\sigma=0.01$, and small rotation rates, $\Omega<8$.

\section{B. Numerical methods}

The system of equations (3) and boundary conditions (4) has been solved numerically using the algorithm described in Ref. [18], which can be summarized as follows. To integrate the equations in time, we use the second-order time-splitting method proposed in Ref. [19] combined with a pseudospectral method for the spatial discretization, Galerkin-Fourier in the azimuthal coordinate $\theta$, and Chebyshev collocation in $r$ and $z$. The radial dependence of the functions is approximated by a Chebyshev expansion between $-R$ and $R$ but forcing the proper azimuthal parity of the variables at the origin $[20,21]$. For instance, the scalar field $\Theta$ and the axial velocity $w$ have an even parity, $\Theta(-r, \theta)=\Theta(r, \theta+\pi)$, whereas $u$ and $v$ are odd functions. To avoid including the origin in the mesh grid, we use an odd number of Gauss-Lobatto points in $r$, and we enforce the equations only in the interval $(0, R]$. We use the standard combination $u_{+}=u+i v$ and $u_{-}=u-i v$ in order to obtain, as a result of the splitting, Helmholtz equations for all the variables $\Theta, w, u_{+}$, and $u_{-}$. For each Fourier mode, these equations are solved using a diagonalization technique in the two coordinates $r$ and $z$. The imposed parity of the functions guarantees the regularity conditions at the origin needed to solve the Helmholtz equations [22]. The same numerical code has been used by the authors and collaborators to study other problems in convection in vertical cylinders, such as binary fluid convection $[23,24]$ and rotating convection $[25,26]$.

Steady solutions have been computed with a Newton's method. We have used a first-order version of the timestepping code described above for the calculation of a Stokes preconditioner that allows a matrix-free inversion of the preconditioned Jacobian needed in each Newton iteration [27]. The corresponding linear system is solved by an iterative technique using a GMRES package [28]. The left-hand side of the preconditioned linear system (Jacobian acting on the correction) corresponds to one time step of the linearized equations, and the right-hand side corresponds to performing one time step of the full nonlinear equations. In this way, the Jacobian matrix is never constructed nor stored [27].

As far as the linear stability analysis of the steady states is concerned, once they have been calculated by the method described before, estimations of eigenvalues and eigenvectors of the linearized problem have been obtained with an Arnoldi's method. The method is applied to calculate the dominant eigenvalues of the exponential of the Jacobian, which can be trivially related to the leading eigenvalues of the Jacobian, i.e., those with the largest real part. To this end, the algorithm for the time stepping of the linearized equations has been used, since, in fact, it approximates the action of the exponential transformation of the Jacobian on the solution at the previous time step. The eigensolving itself has been implemented using the ARPACK package. To determine eigenvalues and eigenvectors accurately, we use the estimated eigenvalues and eigenvectors as the initial guess to solve, via a Newton's method, the nonlinear system (eigenvalues are also unknowns) derived from the eigenvalue problem [29,30]. Once the dominant eigenvalue is identified, a secant method is used to obtain the critical Rayleigh numbers (real part of the eigenvalue equal to zero) and frequencies at the bifurcation points.

In the results reported in the present paper we have used a resolution that ensures variations of the values of Rayleigh number and frequency at the bifurcation points smaller than $0.5 \%$. We have used grids of $n_{r}=32, n_{z}=52, n_{\theta}=52$ points in the radial, axial, and azimuthal directions, respectively, for rotation rates $\Omega \leqslant 1$ and $n_{r}=32, n_{z}=64, n_{\theta}=64$ for higher values. The variations of $0.5 \%$ have been obtained in comparison with results obtained with a grid of $n_{r}=64$, $n_{z}=72, n_{\theta}=72$. In the time integration we have used a time step $\Delta t=5 \times 10^{-4}$. 
(a)
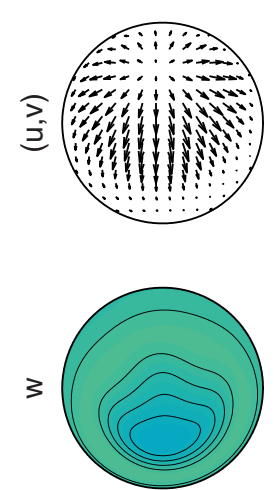

$\mathrm{z}=0.5 \Gamma$
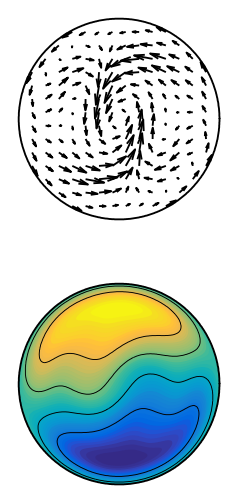

$\mathrm{z}=0.975 \Gamma$
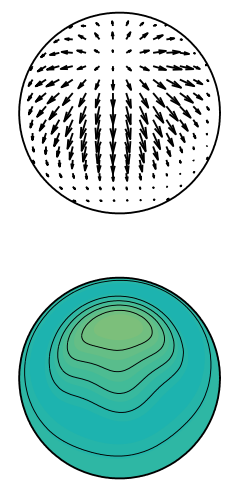

(b)
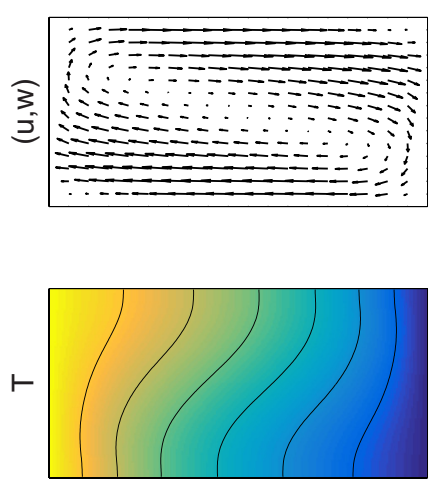

FIG. 2. (a) Transverse flow (top) and contour plots of the axial velocity $w$ (bottom) in the vertical cross sections $z=0.025 \Gamma, z=0.5 \Gamma$, and $z=0.975 \Gamma$. The positive $z$ axis, perpendicular to the paper, points inwards. (b) Longitudinal flow and contour plots of temperature in the plane in which the axial velocity is maximum $(\sigma=0.01, \mathrm{Ra}=4500, \Omega=1.1, \Gamma=2)$.

\section{RESULTS}

\section{A. Symmetries and basic state}

Equations (3), together with boundary conditions (4), are invariant under the transformation operator

$$
\begin{aligned}
& R:(r, \theta, z) \rightarrow(r, \theta+\pi, \Gamma-z), \\
& (u, v, w, \Theta) \rightarrow(u, v,-w,-\Theta),
\end{aligned}
$$

i.e., a point symmetry with respect to the center of the cylinder. Since $R^{2}=I$, the transformation $R$ is a generalized reflection, and the resulting symmetry group is $\mathbf{Z}_{2}=\{I, R\}$.

When expressed in rectangular coordinates, the transformation $R$ acts as follows:

$$
\begin{aligned}
& R:(x, y, z) \rightarrow(-x,-y, \Gamma-z), \\
& \left(v_{x}, v_{y}, v_{z}, \Theta\right) \rightarrow\left(-v_{x},-v_{y},-v_{z},-\Theta\right) .
\end{aligned}
$$

Equivariance of equations and boundary conditions under $R$ means that if $\Psi=(u(r, \theta, z), v(r, \theta, z), w(r, \theta, z), \Theta(r, \theta, z))$
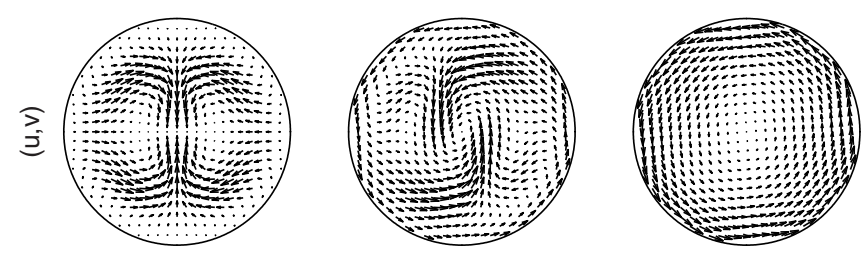

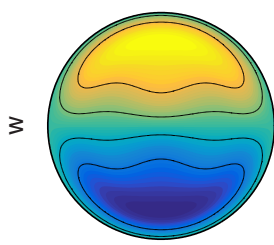

$\Omega=0$

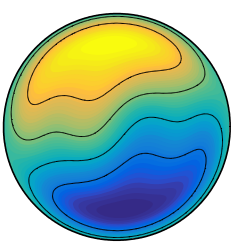

$\Omega=1.1$

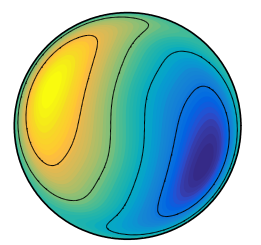

$\Omega=5.1$
FIG. 3. Transverse flow (top) and contour plots of the axial velocity $w$ (bottom) in the central cross section $z=0.5 \Gamma$ for rotation rates $\Omega=0,1.1,5.1$ and Rayleigh number $\mathrm{Ra}=3400$. The positive $z$ axis, perpendicular to the paper, points inwards $(\sigma=0.01, \Gamma=2)$. is a solution, then the field $R \Psi=(u(r, \theta+\pi, \Gamma-z), v(r, \theta+$ $\pi, \Gamma-z),-w(r, \theta+\pi, \Gamma-z),-\Theta(r, \theta+\pi, \Gamma-z))$ is also a solution of the problem. These two solutions are not required to be the same. If so, then we say that the solution possesses the $R$ symmetry, because it is invariant under the transformation $R$. The basic state of this problem, obtained for small values of the Rayleigh number, is a steady three-dimensional solution, invariant with respect to this symmetry.

In Fig. 2 we show the basic state obtained for $\Gamma=2$, Prandtl number $\sigma=0.01$, Rayleigh number $\mathrm{Ra}=4500$, and rotation rate $\Omega=1$.1. In order to make the symmetry $R$ of this solution explicit, we represent in Fig. 2(a) the transverse flow (top) and the contour plots of the axial velocity (bottom) in three cross sections, the central one at $z=0.5 \Gamma$ and the other two equidistant from it, at $z=0.025 \Gamma, 0.975 \Gamma$. For the sake of clarity, we have not used the same scale for the length of the arrows in each figure. For this solution, the maximum transverse velocity in the cross sections at $z=0.025 \Gamma$ and $z=0.975 \Gamma$ is 3.54 , approximately 4.5 times larger than in the $z=0.5 \Gamma$ plane $(0.80)$. Notice that in these cross sections, the maximum transverse velocity is bigger than the azimuthal velocity at the walls, $v=\Omega R / l=0.65$. Due to the rotation of the wall, the reflection invariance with respect to the vertical plane $y=0$ that the basic flow manifested in the case of $\Omega=0$ [12] is now broken, and we observe a tilt in the longitudinal circulation with respect to this plane, as can be appreciated in the contour plots of the axial velocity field. In Fig. 2(b) we plot, on top, the longitudinal circulation flow in the tilted rectangular plane in which the axial velocity is maximum ( $w=5.13)$ and, on bottom, the contour plots of the temperature field $T$ in the same plane. We can observe how this field $T$ is modulated by the advection of the convection currents. Here and hereafter, in all the contour-plot figures, dark areas (blue, when printed in color) represent low intensity and light areas (yellow, in color) correspond to high intensity. Also, hereafter, in the cross-section figures, the $x$ axis points downwards, parallel to gravity, and the $y$ axis points to the left.

The main characteristics of this solution vary when the rotation rate is increased. In Fig. 3 we plot the transverse flow (top) and the contour plot of the axial velocity (bottom) in the cross section $z=0.5 \Gamma$ for different rotation rates, 
(a)

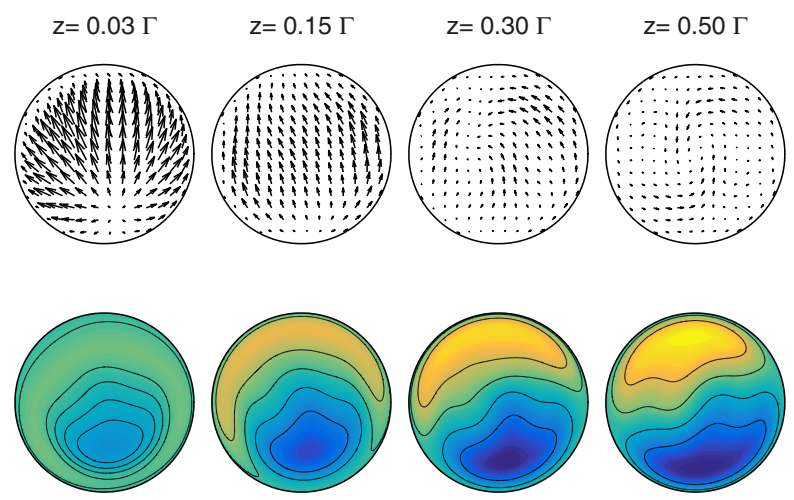

(b)

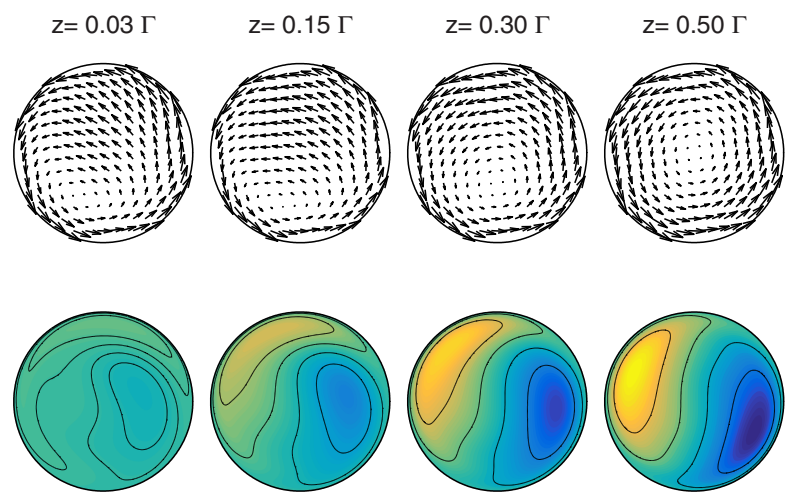

FIG. 4. Transverse flow (top) and contour plots of the axial velocity $w$ (bottom) in different cross-section vertical planes for rotation rates (a) $\Omega=1.1$ and (b) $\Omega=5.1$ and Rayleigh number $\mathrm{Ra}=3400$. The positive $z$ axis, perpendicular to the paper, points inwards $(\sigma=0.01, \Gamma=2)$.

$\Omega=0,1.1,5.1$, and the same value of the Rayleigh number, $\mathrm{Ra}=3400$. To better visualize the flow, again we have not used the same scale for the length of the arrows in each figure. Important changes are observed in the transverse flow in the mid cross section; the flow is partially carried by the movement of the wall for $\Omega=1.1$, while it is strongly dominated by the rotation for $\Omega=5.1$. The tilt of the longitudinal flow can be clearly appreciated in the contour plots of the axial velocity; its value for $\Omega=1.1$ is approximately $6^{\circ}$ with respect to the vertical (negative $y$ axis), whereas for $\Omega=5.1$ it is $62^{\circ}$. This tilt has been evaluated from the value of the azimuthal coordinate of the point with maximum axial velocity.

Additional information about the flows for these solutions can be obtained from Fig. 4, which shows the transverse flow (top) and axial velocity (bottom) in different cross sections for the same value of the Rayleigh number, $\mathrm{Ra}=3400$. In Fig. 4(a) the value of the rotation rate is $\Omega=1.1$, while in Fig. 4(b) it is $\Omega=5.1$. Only cross sections corresponding to the left part of the cylinder are shown; on the right part, the flow is related with that on the left by the transformation $R$.
Notice that we have not used the same scale for the length of the arrows in both solutions, but we have maintained the same scale for the different plots of the same rotation rate. For $\Omega=1.1$, the maximum transverse velocity is 3.01 near the lids of the cylinder and for $\Omega=5.1$ it is 2.55 , i.e., the value corresponding to the azimuthal velocity at the wall. For $\Omega=1.1$, the maximum transverse velocity in the mid cross section is 0.65 . The maximum axial velocity values decrease with $\Omega$, e.g., $w=4.32$ for $\Omega=1.1$ and $w=2.8$ for $\Omega=5.1$, whereas for $\Omega=0$ the maximum axial velocity was 4.39 .

The difference between these flows is attributed to the competition between thermal effects (natural convection) and rotation, which is significant for low rotation rates. This fact can be clearly inferred from Fig. 5(a), which shows the total kinetic energy as a function of the rotation rate. In the region of parameters where these two effects are comparable, the total kinetic energy decreases, despite the increase in the azimuthal flow [see Fig. 5(b)]. This is possible due to the substantial reduction of the longitudinal flow. However, at a certain value of the rotation rate, which becomes larger as the Rayleigh (a)

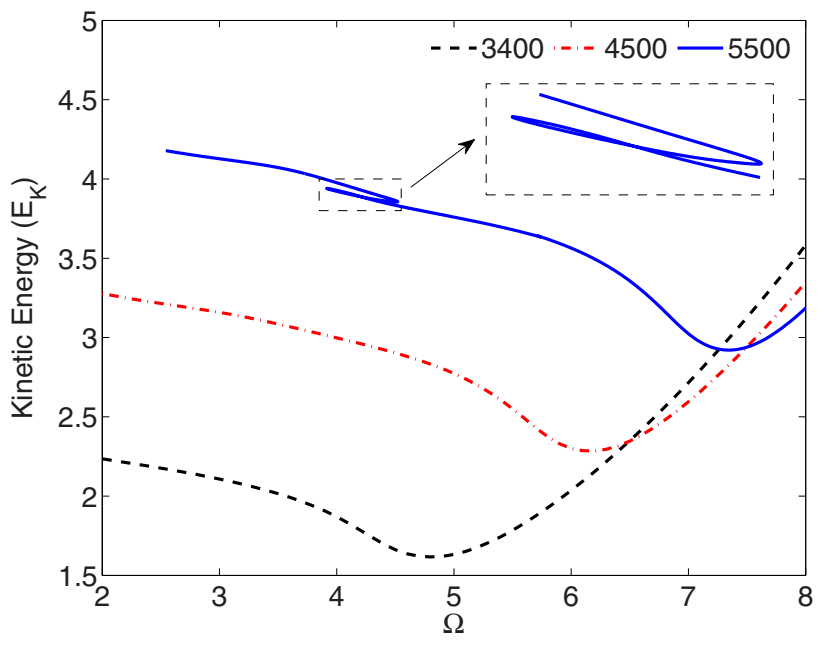

(b)

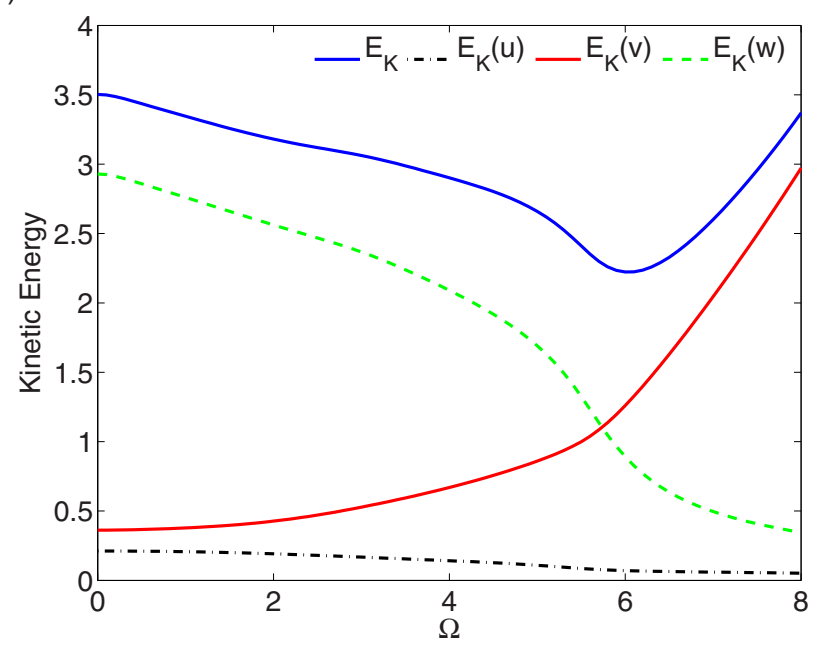

FIG. 5. (a) Mean kinetic energy $E_{k}$ as a function of the rotation rate $\Omega$ for different values of the Rayleigh number Ra $=3400,4500,5500$. (b) Mean kinetic energy associated to each component of the velocity $E_{k}(u), E_{k}(v), E_{k}(w)$ as a function of the rotation rate $\Omega$ for Ra $=4400$. The total mean kinetic energy $E_{k}$ is also plotted $(\sigma=0.01, \Gamma=2)$. 
(a)

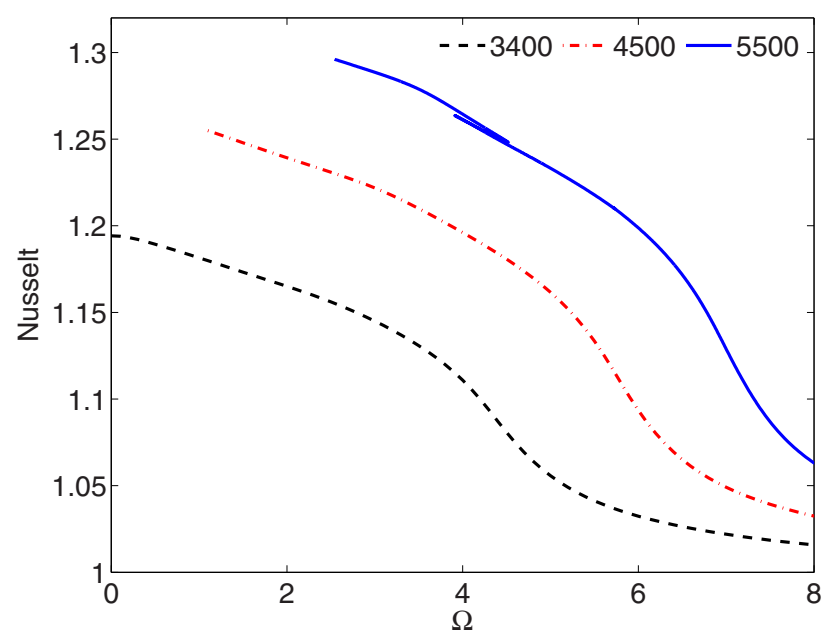

(b)

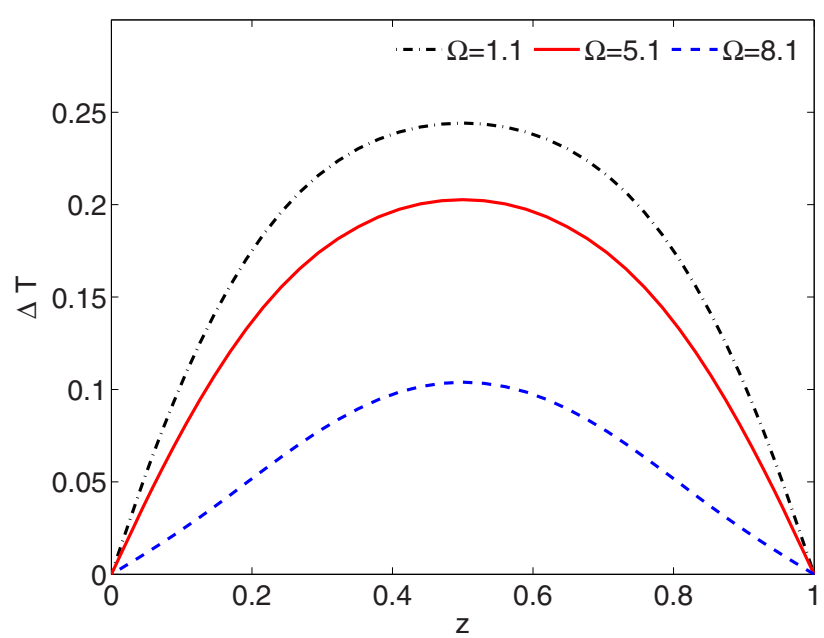

FIG. 6. (a) Nusselt number as a function of the rotation rate $\Omega$ for different values of the Rayleigh number, Ra $=3400,4500,5500$. (b) Difference between the maximum and minimum temperature at every cross section for different values of the rotation rate, $\Omega=1.1,5.1,8.1$, for $\mathrm{Ra}=4400(\sigma=0.01, \Gamma=2)$.

number increases, the azimuthal rotation dominates the flow and the kinetic energy begins to grow. The solution shown in Fig. 4(b) for $\Omega=5.1$ and $\mathrm{Ra}=3400$ is located in the zone of increasing energy and is therefore dominated by the rotation, whereas if we analyze for the same rotation rate the solution for $\mathrm{Ra}=4500$, located in the zone of decreasing energy, we obtain a flow in which the main characteristics of the flow depicted in Fig. 2 can still be identified. In Fig. 6(a), which shows the Nusselt number as a function of the rotation rate, the two zones in each curve are separated by an inflection point where the sign of the curvature changes. Rotation slows down the heat transport in both zones, regardless of whether rotation dominates or not.

When the rotation rate increases, the difference between the extreme values of temperature decreases, giving rise to a

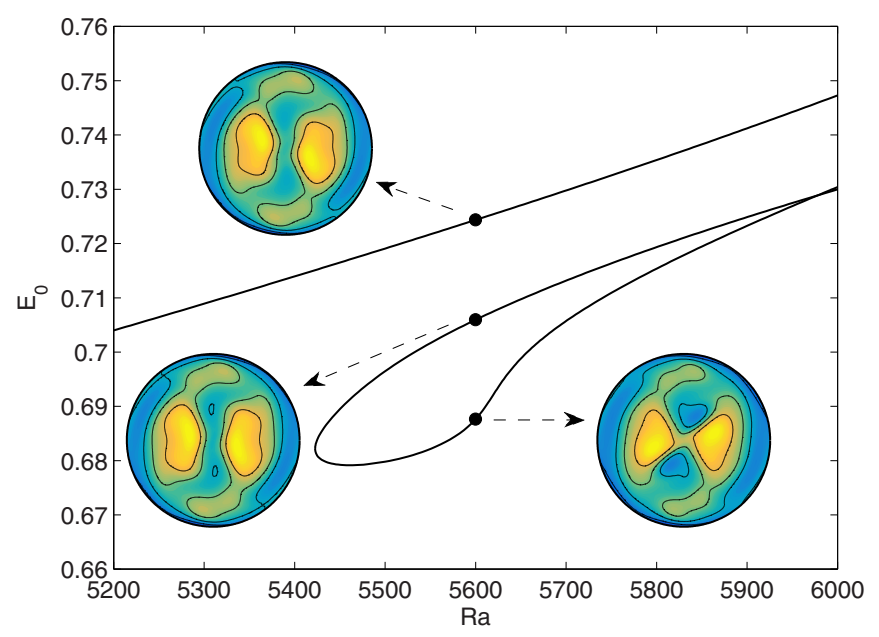

FIG. 7. Main and disconnected curves of basic solutions showing the kinetic energy associated to the zero azimuthal modes, $E_{0}$, as a function of the Rayleigh number for $\Omega=4.4$, and contour plots of axial vorticity at $z=0.5 \Gamma$ for the three different basic solutions that exist at $\mathrm{Ra}=5600(\sigma=0.01, \Gamma=2)$. homogenization of temperature related to the attenuation of the longitudinal flow. This fact can be observed in Fig. 6(b), where this difference in every cross section is plotted for three values of the rotation rate, $\Omega=1.1,5.1,8.1$, and a Rayleigh number of $\mathrm{Ra}=4500$. Only for the last value of $\Omega$ is the solution dominated by rotation.

A careful inspection of Fig. 5(a) reveals a crucial detail: for $\mathrm{Ra}=5500$ and $\Omega \approx 4$ there is a zone where three solutions with the same value of the Rayleigh number and the rotation rate have different total kinetic energies. This situation corresponds to the existence of two different curves of steady $R$-invariant solutions when rotation is fixed and the Rayleigh number varies. These two different curves of basic solutions can be visualized in Fig. 7, which shows a global quantity (amplitude), representative of the solution, as a function of the Rayleigh number for a rotation rate $\Omega=4.4$. The amplitude chosen for representing the solutions is $E_{0}$, which is the contribution of the zero azimuthal modes to the kinetic energy, defined as

$$
E_{0}=\frac{\int_{0}^{H / l} \int_{0}^{R / l}\left(\left|\hat{u}_{0}\right|^{2}+\left|\hat{v}_{0}\right|^{2}+\left|\hat{w}_{0}\right|^{2}\right) r d r d z}{(R / l)^{2}(H / l)} .
$$

Hereafter, to identify these two curves of basic solutions, we will name the curve of solutions that extends towards small values of the Rayleigh number for a fixed rotation rate main and the curve with two branches originating from a saddle node disconnected.

In the same figure, we include three insets showing the contour plots of the axial vorticity in the central cross section of the three solutions for $\mathrm{Ra}=5600$. The use of vorticity in the representation enhances the differences between the different flows. Positive values of vorticity [light (yellow) parts] correspond to a clockwise rotating vortex, while negative values [dark (blue) parts] are associated to an counterclockwise rotating vortex. As can be appreciated, two of these three insets look very similar, their inner part consists of two elongated clockwise vortices, whereas four vortices can be 
(a)

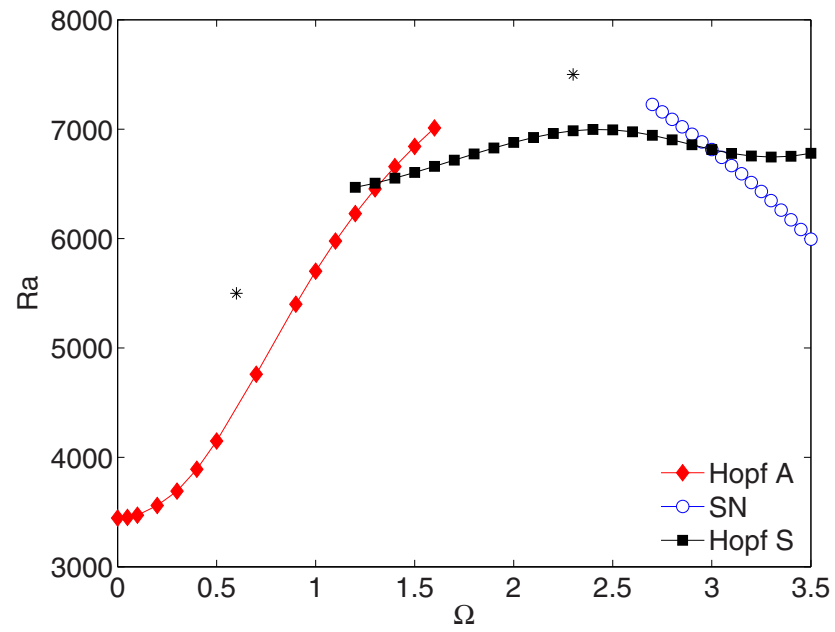

(b)

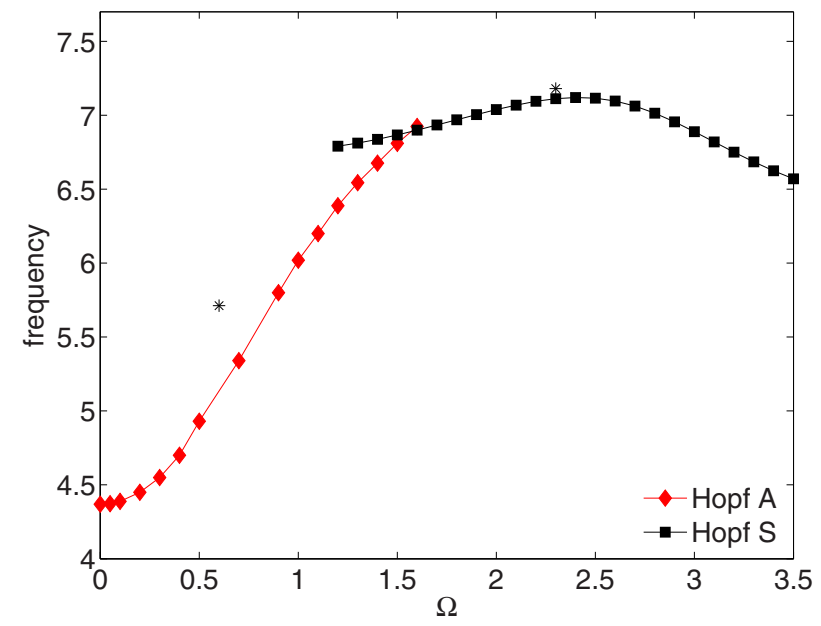

FIG. 8. (a) Critical Rayleigh number and (b) the corresponding angular frequency of the primary instability of the basic flow (Hopf bifurcation) as a function of the rotation rate. The line with solid diamonds (red) corresponds to Hopf bifurcations that break the $R$ symmetry, the line with solid squares (black) corresponds to Hopf bifurcations that preserve the $R$ symmetry, and the line with open circles (blue) denotes the position of the saddle-node bifurcations that limit the zone in which three branches of steady $R$-invariant solutions coexist $(\sigma=0.01, \Gamma=2)$.

easily identified in the third inset. In fact, the two branches to which the alike solutions belong will connect for a higher value of the rotation rate $\Omega$, as will be explained later.

The stability of the solutions in these three branches will be analyzed in the next section as a function of the Rayleigh number for a range of values of rotation rates from $\Omega=0$ to $\Omega=4.6$, that is, the region in which the flow is not dominated by rotation yet. The position of the saddle node points limits the region in which the three branches of solutions exist.

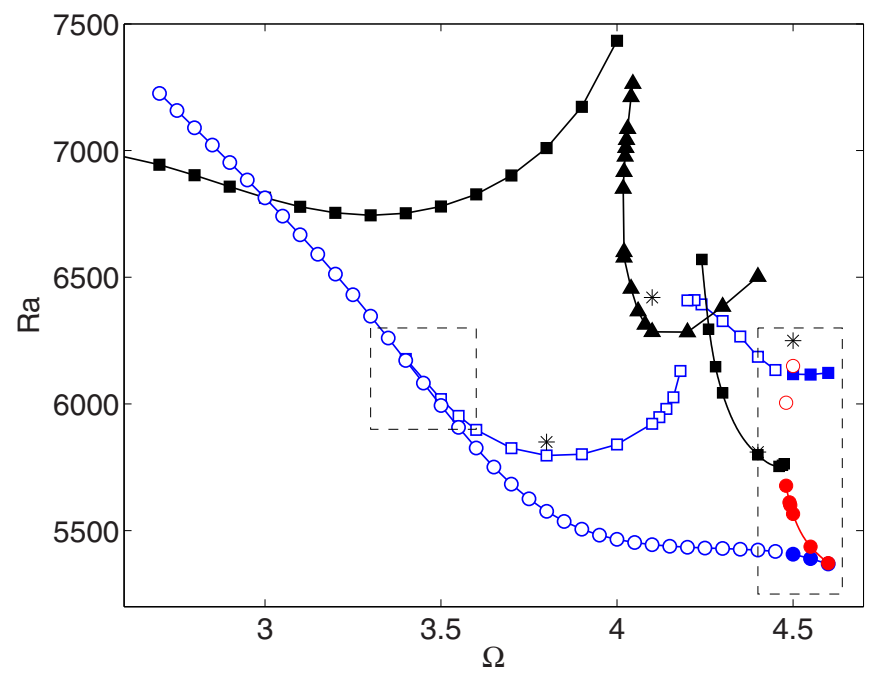

FIG. 9. Extended bifurcation diagram showing the critical Rayleigh number as a function of the rotation rate. The basic flows whose stability is analyzed are steady and $R$ invariant. Circles denote saddle-node points, squares Hopf bifurcations, and triangles pitchfork bifurcations. Solid symbols are used for bifurcations of the solutions in the main curve and open symbols for bifurcations of solutions belonging to the disconnected curve. The two regions delimited by dashed rectangles will be discussed in detail in next figures $(\sigma=0.01, \Gamma=2)$.

\section{B. Linear stability analysis of the basic flow}

In this section we present the results corresponding to the linear stability analysis of the steady $R$-symmetric solutions belonging to the two curves that we have identified for rotation rates in the range $\Omega \in(0,4.6)$. As is well known in this problem, the instabilities of the symmetric basic steady state can be (i) steady or oscillatory, breaking the $R$ symmetry (with antisymmetric eigenvector with respect to the broken symmetry), or (ii) oscillatory, maintaining the symmetry. Obviously, a saddle-node bifurcation (a steady bifurcation maintaining the $R$ symmetry) is also possible.

For very small rotation rates, $0<\Omega<3.5$, the stability analysis of the basic flow reveals that the primary instabilities are Hopf bifurcations; the corresponding critical Rayleigh numbers and angular frequencies are shown in Fig. 8 as a function of $\Omega$. Two different types of instabilities are obtained. The line with solid diamonds (red) corresponds to Hopf bifurcations that break the $R$ symmetry and for which rotation has an important stabilizing effect. The line with solid squares (black) is also a Hopf bifurcation, but in this case the $R$ symmetry is preserved and, as can be seen in Fig. 8, an increase of $\Omega$ hardly affects the stabilization of the flow. The transition between these two Hopf bifurcations occurs at $\Omega \approx 1.35$. The line with open circles (blue) indicates the location of the saddle-node bifurcations that limit the zone in which the three branches of steady $R$-invariant solutions exist. As we will discuss later, above this line, for $\Omega \lesssim 3.4$ the two branches of the disconnected curve are unstable. The points marked with an asterisk in this figure correspond to the values of parameters in which secondary flows will be shown.

The extension of the bifurcation diagram to higher rotation rates can be seen in Fig. 9, where the critical Rayleigh number at which the steady $R$-invariant solutions involved become unstable has been plotted as a function of the rotation rate. Only the dominant instabilities have been included in this figure. Circles denote saddle-node points, squares Hopf bifurcations that maintain the $R$ symmetry, and triangles pitchfork 
bifurcations that break the $R$ symmetry of the basic flow. Solid symbols denote bifurcations of the basic flow belonging to the main curve and open symbols denote bifurcations of solutions belonging to the disconnected curve. The two regions delimited by dashed rectangles will be discussed in detail further below. The open-circled line (blue) limits the region in which the three branches of steady $R$-invariant solutions exist. The curve with open squares (blue) corresponds to Hopf bifurcations of the solutions belonging to one of the branches of the disconnected curve. These Hopf bifurcations maintain the $R$ symmetry and have a low value of frequency. The discontinuity observed in the curve with open squares (blue) near $\Omega \approx 4.2$ is caused by the cubic dependence with the Rayleigh number of the real part of the eigenvalue responsible for the instability. The maximum value of this cubic curve is zero for the value of $\Omega$ where the discontinuity occurs. Solutions in the other branch of the disconnected curve are unstable. More details about the stability of the disconnected curve will be given below, when we describe the zones limited by the dashed rectangles near $\Omega=3.45$ and $\Omega=4.5$.

Still in Fig. 9, as far as the stability of the solutions in the main curve is concerned, we plot the prolongation of the solid-squared line (black) in Fig. 8, which corresponds to the Hopf bifurcation that maintains the $R$ symmetry. This bifurcation is responsible for the instability in the range $1.33 \leqslant \Omega \leqslant 4$. For $4<\Omega<4.3$, the dominant instability is a pitchfork bifurcation that breaks the $R$ symmetry and is depicted with a line of solid triangles (black). At a higher value of the rotation rate, $\Omega \approx 4.25$, this instability is superseded by a low-frequency Hopf bifurcation that maintains the $R$ symmetry of the basic flow and that shows a steep decrease in the associated critical Rayleigh number when $\Omega$ increases. This instability is denoted by a solid-squared line (black). The region limited by the dashed rectangle near $\Omega=4.5$, where solid and open circles (red) and solid and open squares (blue) appear, deserves special focus and will be discussed below.

Once we have identified the dominant instabilities in the range of $\Omega$ we are considering, we are going to discuss in more detail the stability properties of the basic solutions around $\Omega=3.45$ and $\Omega=4.5$, that is, in the regions delimited by dashed rectangles in Fig. 9. In both cases, to help the understanding of these results, we use schematic pictures in which the more representative bifurcation points and the sign of the corresponding eigenvalues are shown. The schemes in Fig. 10(a) show the disconnected curve for fixed values of the rotation rates around $\Omega=3.45$ and those in Fig. 11(a) show the main and the disconnected curves for rotation rates around $\Omega=4.5$. In Fig. 10(a) we deal with bifurcations of solutions in the disconnected curve, so the symbols used to denote the bifurcation points are open symbols, while in Fig. 10(b) both solid and open symbols are used to identify whether solutions belong to the main curve or to the disconnected one. In these schemes, squares denote a Hopf bifurcation that maintains the $R$ symmetry, circles denote a saddle-node bifurcation, and diamonds denote a new Hopf bifurcation, which breaks the $R$ symmetry and was not included in Fig. 9. The sign of the eigenvalues associated to the bifurcations in the different regions of the schematic branches are plotted in two rows: The top (red) row corresponds to the Hopf bifurcation that breaks the $R$ symmetry (diamond), and the bottom (black) row corresponds to bifurcations that maintain the $R$ symmetry (square and circle). Two equal signs in brackets represent a pair of complex conjugates. In all the schemes, the Rayleigh number is in the horizontal axis, increasing towards the right. Thick lines correspond to stable solutions.

The transitions identified in the first zone, around $\Omega=$ 3.45 , when the rotation rate decreases are the following. At $\Omega=3.45$ the Hopf bifurcation that breaks the $R$ symmetry (diamond) is located in one of the branches of the disconnected (a)
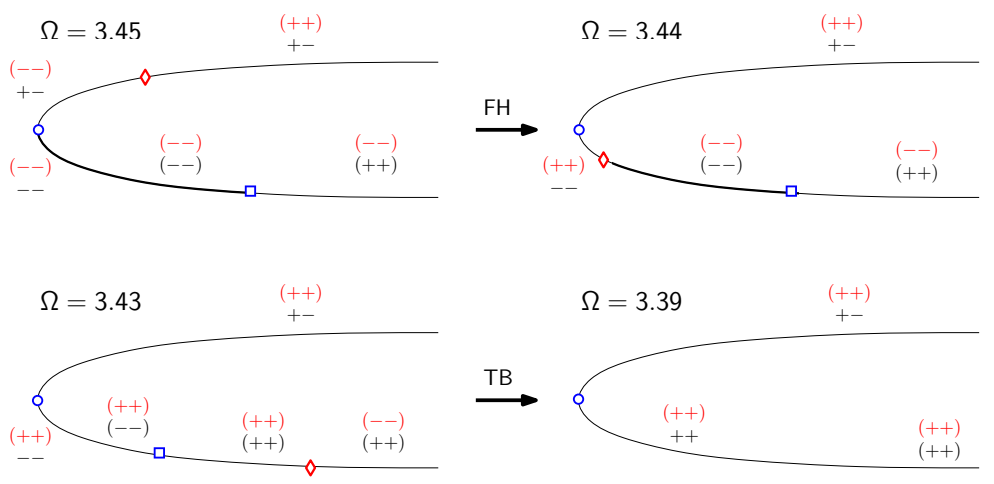

(b)

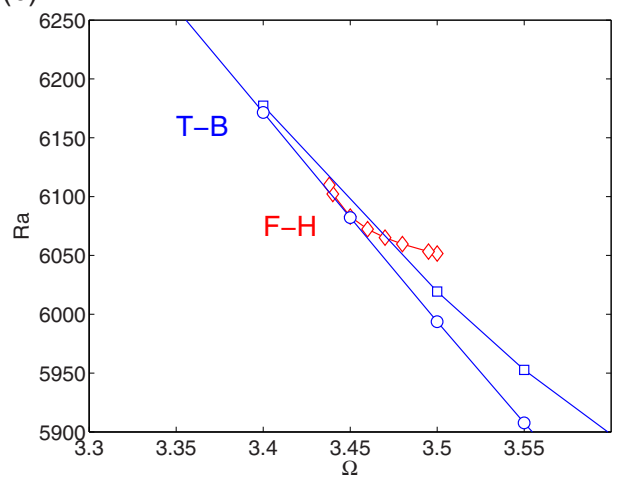

FIG. 10. (a) Schematic pictures showing the disconnected curve for fixed values of the rotation rates, $\Omega=3.45,3.44,3.43,3.39$. Squares (blue) denote low-frequency Hopf bifurcations that maintain the $R$ symmetry, diamonds (red) denote Hopf bifurcations that break the $R$ symmetry, and circles (blue) denote saddle-node bifurcations. The signs of the eigenvalues associated to the bifurcations in the different regions of the schematic branches are plotted in two rows: The top (red) row corresponds to the Hopf bifurcation that breaks the $R$ symmetry (diamond) and the bottom (black) row corresponds to the Hopf bifurcation that maintains the $R$ symmetry (square). Two equal signs in brackets represent a pair of complex conjugates. Thick lines indicate that solutions on this part of the branches are stable. (b) Actual bifurcation diagram showing the location of the Hopf bifurcation that preserves the $R$ symmetry (line with blue squares), the Hopf bifurcation that breaks the $R$ symmetry (line with red diamonds), and the saddle-node points of the disconnected curve (line with blue circles) in the ( $\Omega$, Ra) parameter space $(\sigma=0.01, \Gamma=2)$. 
(a) $\Omega=4.47$

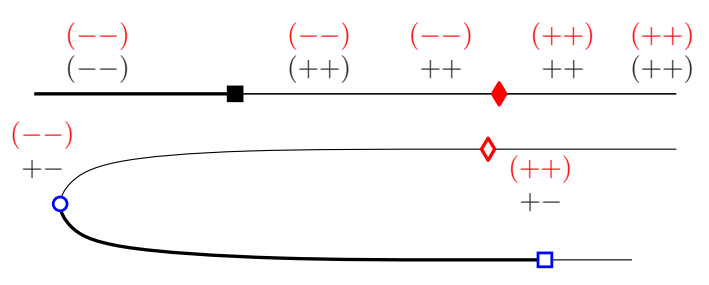

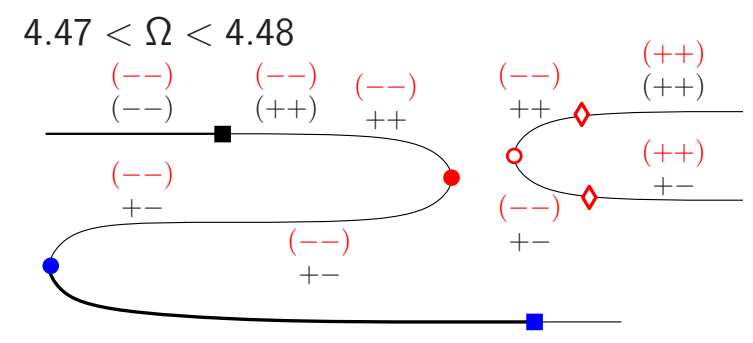

$\Omega=4.6$
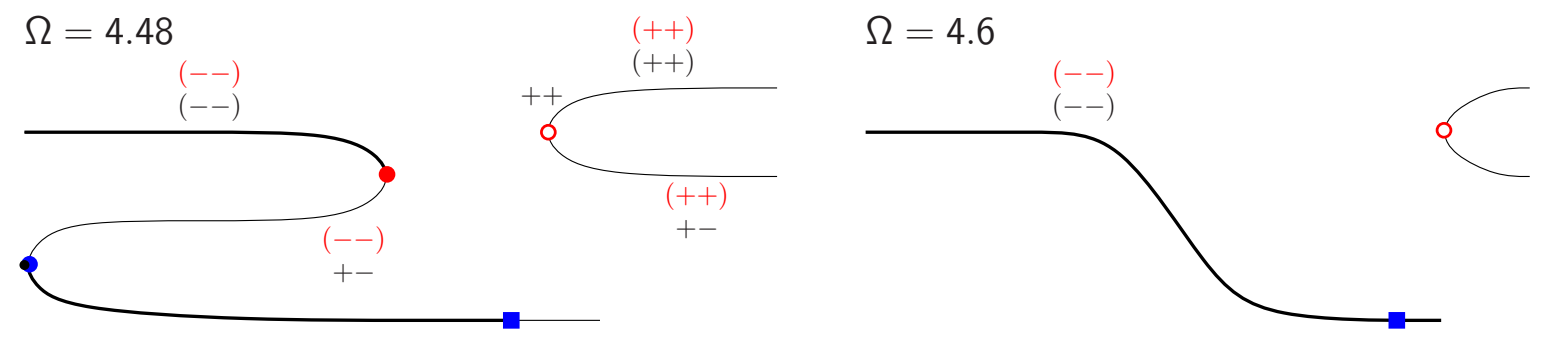

(b)

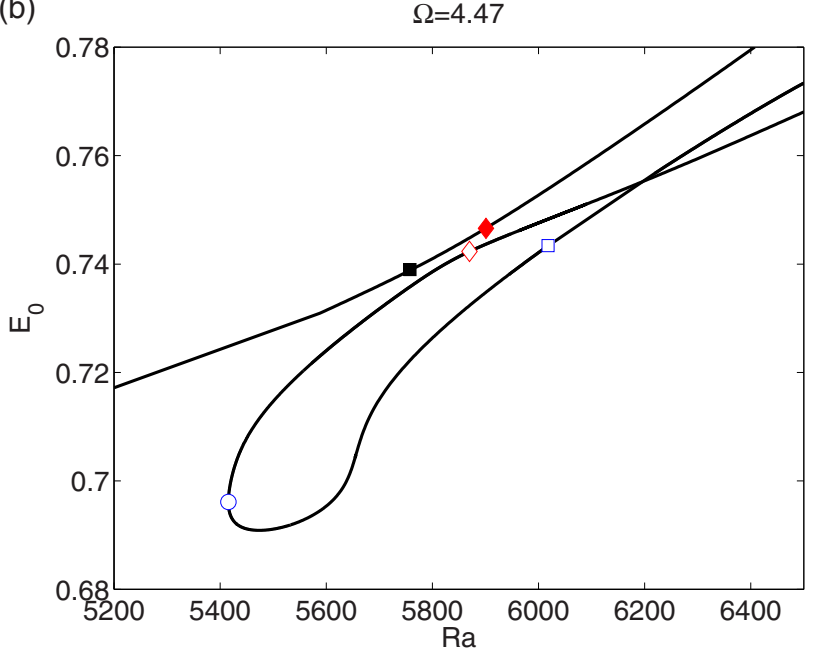

(c)

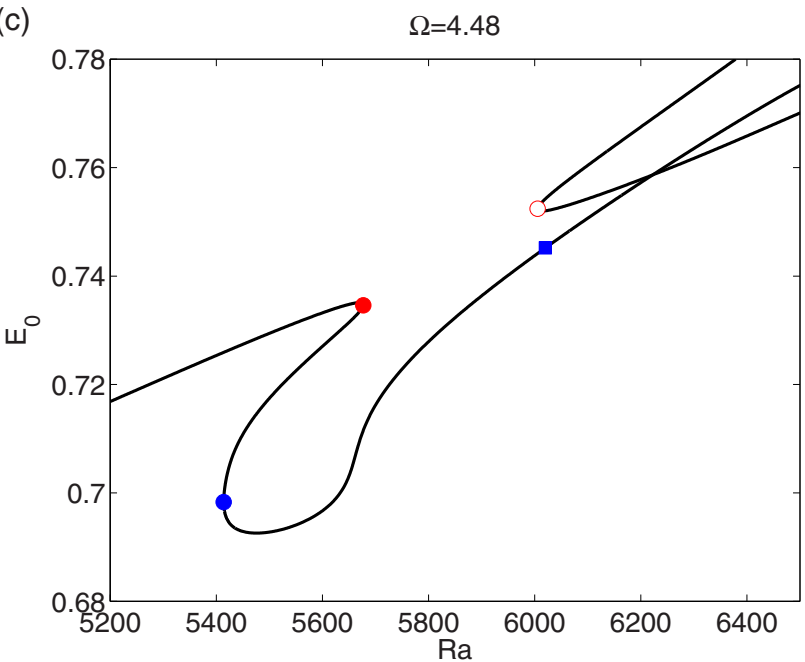

FIG. 11. (a) Schematic pictures showing the disconnected curves for fixed values of the rotation rates: $\Omega=4.47$, an unspecified value of $\Omega$ between 4.47 and $4.48, \Omega=4.48$, and $\Omega=4.6$. Squares (blue and black) denote Hopf bifurcations that maintain the $R$ symmetry, diamonds (red) denote Hopf bifurcations that break the $R$ symmetry, and circles (red and blue) denote saddle-node points. The signs of the eigenvalues associated to the bifurcations that maintain the $R$ symmetry (squares and circles) and to the $R$-symmetry-breaking bifurcation (diamonds) in the different regions of the schematic branches are plotted in two rows: (top) those corresponding to the Hopf bifurcation that breaks the $R$ symmetry (diamonds) and (bottom) those corresponding to the bifurcations that maintain the $R$ symmetry (squares and circles). Two equal signs in brackets represent a pair of complex conjugates. Thick lines correspond to stable solutions. Actual bifurcation diagrams for (b) $\Omega=4.47$ and (c) $\Omega=4.48$, showing the zero azimuthal contribution to the kinetic energy $E_{0}$ of the solutions versus the Rayleigh number. The position of the three Hopf bifurcations and of the saddle-node points is denoted with the same symbols used in the schematic pictures $(\sigma=0.01, \Gamma=2)$.

curve [the upper branch in the scheme in Fig. 10(a)], while the Hopf bifurcation that breaks the $R$ symmetry, which has a low value of frequency (square), is in the other branch (lower branch). Between the the saddle-node point and the low-frequency Hopf bifurcation there is a region where the basic solutions are stable. From $\Omega=3.45$ to $\Omega=3.44$ a fold Hopf bifurcation involving the Hopf bifurcation that breaks the $R$ symmetry (diamond) takes place, resulting in a reduction of the stable zone. At the same time, the low-frequency Hopf bifurcation (square) approaches the saddle-node point decreasing the frequency. When $\Omega$ decreases from $\Omega=3.44$ to $\Omega=3.43$, the symmetry-breaking Hopf bifurcation (diamond) shifts towards higher values of the Rayleigh number, and the symmetry-preserving Hopf bifurcation (square) shifts towards the saddle-node point, further decreasing its frequency. Since the two Hopf bifurcations interchange positions, the stable region disappears. When $\Omega$ is decreased from $\Omega=3.43$ to $\Omega=3.39$, the low-frequency Hopf bifurcation that maintains the $R$ symmetry (square) disappears in a Takens-Bogdanov bifurcation. Notice that in these four schemes several transitions from two reals to a pair of complex conjugates or the other way around take place; although they have been depicted in the schemes, they do not represent bifurcation points and will not be commented on. The actual bifurcation diagram 
showing the stability in this region is plotted in Fig. 10(b). The position of the two Hopf bifurcations and the saddle-node points is indicated with the same symbols used in the schematic pictures.

Finally, we are going to discuss the results corresponding to the stability analysis of the basic solutions in the region near $\Omega=4.5$, limited by the second dashed rectangle in Fig. 9 when the rotation rate increases. The two curves of basic solutions (main and disconnected curves) change stability in this zone, giving rise to a complex bifurcation diagram. The schemes in Fig. 11(a) correspond to four fixed values of rotation: $\Omega=4.47$, an unspecified value of $\Omega$ between 4.47 and $4.48, \Omega=4.48$, and $\Omega=4.6$. At $\Omega=4.47$, there are two Hopf bifurcations in the main curve of basic solutions. The dominant bifurcation is a low-frequency Hopf bifurcation that maintains the $R$ symmetry (solid black square) and is followed by a Hopf bifurcation that breaks the $R$ symmetry with critical frequency $\omega_{c} \approx 2.5$ (solid diamond). One of the branches of the disconnected curve (the lower branch in the scheme in Fig. 11(a) is stable from the saddle node to the Hopf bifurcation that maintains the $R$ symmetry (open blue square). If this bifurcation was continued by decreasing the rotation rate, then we would obtain the Hopf bifurcation we had identified in the zone $\Omega \approx 3.4$ and that was represented in Fig. 10(a) (open blue square). Solutions in the other branch of the disconnected curve (upper part), near the saddle node, have a positive and a negative real eigenvalue associated to eigenvectors that preserve the $R$ symmetry. When the Rayleigh number increases, a Hopf bifurcation that breaks the $R$ symmetry, with critical frequency $\omega_{c} \approx 2.5$, occurs (open diamond). Therefore, this branch is always unstable.

Between $\Omega=4.47$ and a value smaller than $\Omega=4.48$, a codimension- 2 bifurcation occurs. At some point $(\Omega, \mathrm{Ra})$ between the two Hopf bifurcations, the main curve merges with the unstable branch of the disconnected curve, and two saddle nodes (solid and open red circles) appear. These are the saddle nodes that were represented in Fig. 9. This codimension-2 bifurcation in the literature is called a transcritical or a branching bifurcation. It is worth noticing that the eigenvalues shown in the schematic figure for $\Omega=4.47$ are compatible with the observed transition, provided that the pair of complex conjugates eigenvalues arising after the low-frequency Hopf bifurcation (solid black square) become two real numbers, and one of them decreases enough to change sign in the new saddle-node bifurcations.

Between this intermediate value of $\Omega$ and $\Omega=4.48$, several codimension-2 bifurcations occur. The low-frequency Hopf bifurcation that maintains the $R$ symmetry (solid black square) shifts towards the new saddle-node (solid red circle) decreasing frequency, until disappearing in a Takens-Bogdanov bifurcation. In the new disconnected curve, a fold bifurcation involving one of the Hopf bifurcations that break the $R$ symmetry (open red diamond) takes place, followed by the merging and disappearance of these two bifurcations. At the same time, this new disconnected curve towards higher values of the Rayleigh number. Near $\Omega=4.6$, the two saddle nodes (solid red and blue circles) of the new main curve merge and disappear in a codimension- 2 cusp bifurcation. Figures 11(b) and 11(c) show the actual bifurcation diagrams for $\Omega=4.47$ and $\Omega=4.48$. The position of the three Hopf bifurcations and of the saddle-node points is denoted with the same symbols as those used in the schematic pictures in Fig. 11(a).

\section{Energy analysis of the instability modes}

In the previous section we have analyzed in detail the stability of the steady $R$-invariant solutions (basic flow), both in the case that, for a fixed $\Omega$, they belong to the main curve, which extends to small values of the Rayleigh number, and when they belong to the disconnected curve of solutions. Two different modes of instability can appear, depending on whether the $R$ symmetry of the basic flow is preserved. All the bifurcations that have been identified are supercritical in all the cases that have been considered. In this section, we perform an energy analysis that allows us to characterize the physical mechanisms of each instability, as we had done in a previous work in a nonrotating cylinder [12].

To gain some insight in the physical mechanisms involved in the instabilities we are analyzing, we undergo a kinetic energy transfer analysis at some critical points [3,4,31]. We use the Reynolds-Orr equation obtained by integrating over the volume occupied by the fluid the inner product of the momentum equation for the perturbation and the perturbation of the velocity (critical eigenvector). If we denote by $\left[\mathbf{U}_{\mathbf{0}}, T\right]$ the basic state and by $\left[\mathbf{u}^{\prime}, \Theta^{\prime}\right]$ the eigenvector, then the kinetic energy of the perturbation, defined as $K=1 / 2 \int_{\Omega} \mathbf{u}^{\prime} \cdot \mathbf{u}^{\prime *} d \Omega$, satisfies

$$
\begin{aligned}
\frac{\partial K}{\partial t}= & -\operatorname{Re}\left[\int_{\Omega} \mathbf{u}^{\prime *} \cdot\left(\mathbf{u}^{\prime} \cdot \nabla \mathbf{U}_{\mathbf{0}}\right) d \Omega\right] \\
& -\operatorname{Re}\left[\sigma \int_{\Omega}\left(\nabla \times \mathbf{u}^{\prime}\right) \cdot\left(\nabla \times \mathbf{u}^{\prime *}\right) d \Omega\right] \\
& -\operatorname{Re}\left(\operatorname{Ra} \sigma \int_{\Omega} \mathbf{u}^{\prime *} \cdot \Theta^{\prime} \hat{\mathbf{x}} d \Omega\right) .
\end{aligned}
$$

The first term on the right-hand side represents the production of energy by shear; the second one the viscous dissipation, which is always negative; and the third one the production of energy by buoyancy. The terms with positive (negative) sign destabilize (stabilize) the basic flow. Since the term on the left-hand side is zero at the critical point, the shear term and the buoyancy term must balance the viscous dissipation. If we normalize the right-hand side terms with the absolute value of the viscous dissipation, then the sum of shear and buoyancy terms must be 1 [4]. In Table I we show the critical Rayleigh number, the critical frequency, and the rotation rate at different critical points, together with the symmetric $(\mathrm{S})$ or nonsymmetric (NS) character of the instability, indicating also if the basic flow corresponds to the main curve of solutions (M) or if it belongs to the disconnected curve (D). The values of the normalized shear term in these critical points are also shown. The production of the kinetic energy of the perturbation in all these bifurcations comes clearly from the shear term (normalized shear larger than one). The buoyancy term, as expected, decreases notably when the rotation rate increases but is always negative (stabilizing effect). The contribution of each term to the shear depends on the instability. We also indicate in Table I the main destabilizing parts of the shear term expansion in each case. In all cases, except for $\Omega=2.3$, the main contribution is associated to the term $w^{\prime *} \frac{v^{\prime}}{r} \frac{\partial W_{0}}{\partial \theta}$, although for $\Omega=0.6,2.3,4$.1 the mean value of the destabilizing terms 
TABLE I. Symmetry character of the instability (symmetric S and nonsymmetric NS) and curve (M for solutions belonging to the main curve that extends to small values of the Rayleigh number and D for the disconnected curve), critical Rayleigh number $\mathrm{Ra}_{c}$ and frequency $\omega_{c}$, rotation rate $\Omega$, normalized production of kinetic energy by shear associated with the eigenvectors at some bifurcation points, and main destabilizing term of the shear expansion in each case $(\sigma=0.01, \Gamma=2)$.

\begin{tabular}{|c|c|c|c|c|c|}
\hline Symmetry \& branch & Critical Rayleigh number & Critical frequency & Rotation rate & Normalized shear & Shear term \\
\hline $\mathrm{NS}, \mathrm{M}$ & $4.43 \times 10^{3}$ & 5.19 & 0.6 & 1.036 & $w^{\prime *} \frac{v^{\prime}}{r} \frac{\partial W_{0}}{\partial \theta}$ \\
\hline $\mathrm{S}, \mathrm{M}$ & $6.99 \times 10^{3}$ & 7.11 & 2.3 & 1.013 & $w^{\prime *} u^{\prime} \frac{\partial W_{0}}{\partial r}$ \\
\hline $\mathrm{NS}, \mathrm{M}$ & $6.29 \times 10^{3}$ & 0 & 4.1 & 1.0086 & $w^{\prime *} \frac{v^{\prime}}{r} \frac{\partial W_{0}}{\partial \theta}$ \\
\hline $\mathrm{S}, \mathrm{M}$ & $5.80 \times 10^{3}$ & 0.49 & 4.4 & 1.016 & $w^{\prime *} \frac{v^{\prime}}{r} \frac{\partial W_{0}}{\partial \theta}$ \\
\hline$S, D$ & $5.79 \times 10^{3}$ & 1.13 & 3.8 & 1.0085 & $w^{\prime *} \frac{v^{\prime}}{r} \frac{\partial W_{0}}{\partial \theta}$ \\
\hline $\mathrm{S}, \mathrm{M}$ & $6.12 \times 10^{3}$ & 1.64 & 4.5 & 1.0080 & $w^{\prime *} \frac{v^{\prime}}{r} \frac{\partial W_{0}}{\partial \theta}$ \\
\hline
\end{tabular}

$w^{\prime *} u^{\prime} \frac{\partial W_{0}}{\partial r}$ and $w^{\prime *} \frac{v^{\prime}}{r} \frac{\partial W_{0}}{\partial \theta}$ has the same order of magnitude. The maximum value of these destabilizing terms is always located near the center of the cylinder, away from the boundaries, indicating that the instability is originated in the bulk of the fluid and not in the boundary layers at the container walls. It is also worth mentioning the strong stabilizing contribution of the term $w^{\prime *} w^{\prime} \frac{\partial W_{0}}{\partial z}$ to the instability that takes place at $\Omega=4.1$.

\section{Secondary flows}

In the following, after having discussed the results corresponding to the energy analysis of the instabilities, we describe some of the saturated states of different nature that the system selects once the threshold of the instability is crossed for a choice of rotation rates in the range $\Omega \in(0,4.6)$. These states are representative of the different dominant instabilities and basic flows observed in the system; asterisks are used in Fig. 8 (a) and Fig. 9 to locate them in the $(\Omega$, Ra) parameter space. The procedure we follow to obtain the saturated states consists of setting a slightly supercritical Rayleigh number for which the time-dependent governing equations are numerically integrated until a secondary flow is reached. We take as initial condition the basic flow below onset. Even though most of the secondary flows that we will present are solutions arising from bifurcations that take place in the main curve of primary solutions, we will also describe a saturated solution originated from an instability in the disconnected curve of primary solutions and a solution arising from an instability in the main curve after the reconnection with the disconnected curve has taken place, as described in Fig. 11.

We begin by presenting four secondary solutions obtained for rotation rates $\Omega=0.6,2.3,4.1,4.4$, which arise from instabilities that take place in the main curve of primary solutions.

At a rotation rate of $\Omega=0.6$ the basic steady flow undergoes a symmetry-breaking oscillatory bifurcation at

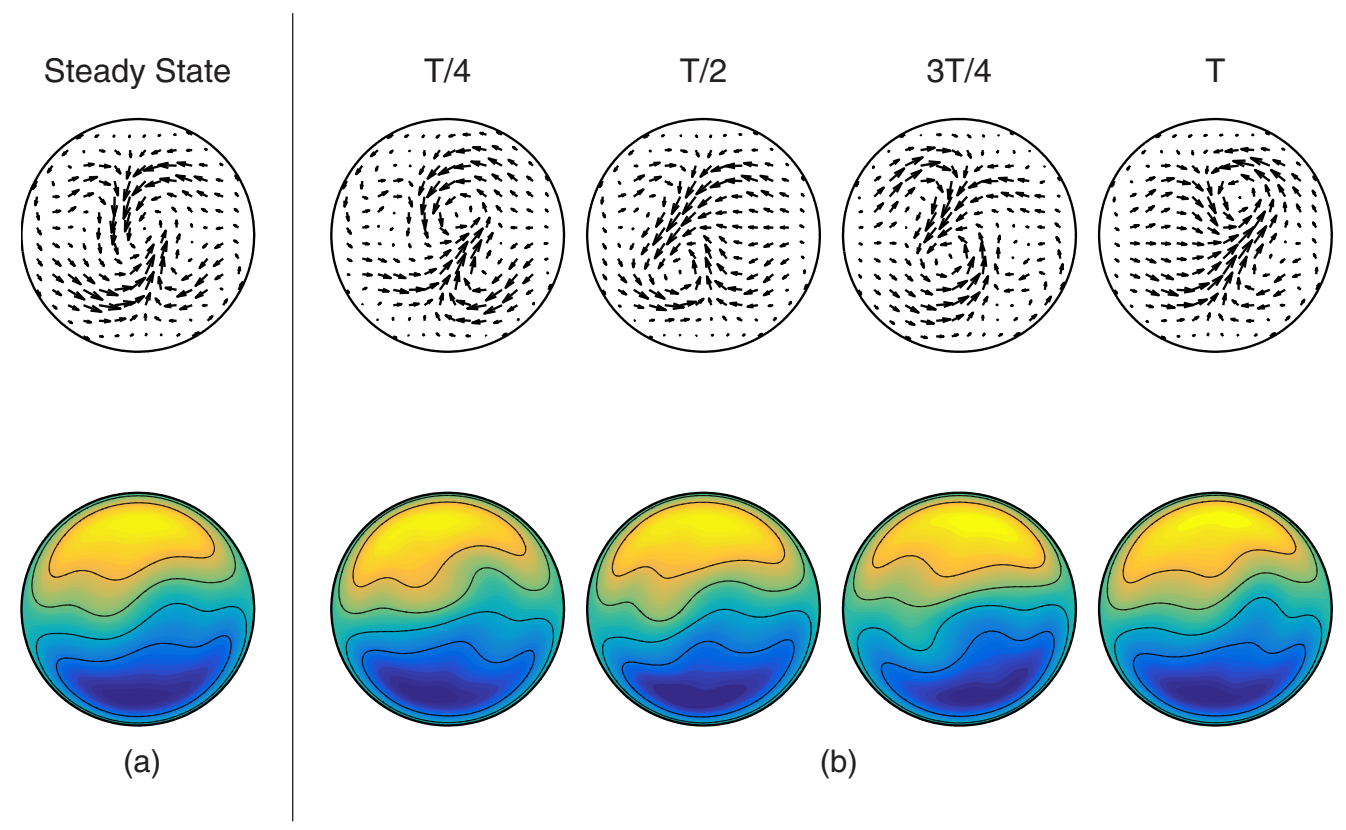

FIG. 12. Nonsymmetric oscillatory flow for $\Omega=0.6$ arising from an instability in the main curve. The cross sections at $z=0.5 \Gamma$ correspond to the transverse velocity field and the contour plots of axial velocity for (a) the basic state and (b) for the oscillatory secondary solution ( $T=1.1$ ) in four time instants obtained at $\mathrm{Ra}=5500$. 


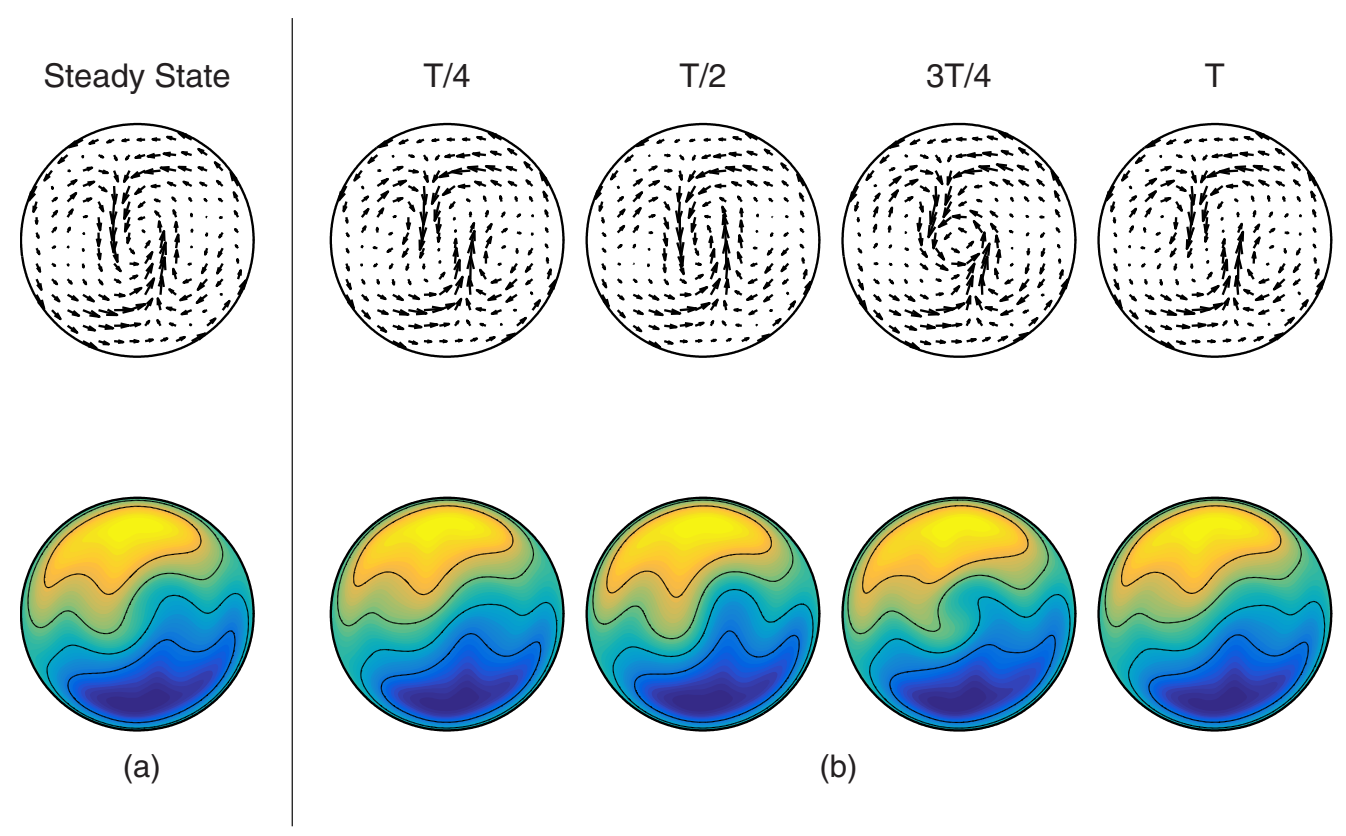

FIG. 13. Symmetric oscillatory flow for $\Omega=2.3$ arising from an instability in the main curve. The cross sections at $z=0.5 \Gamma$ correspond to the transverse velocity field and the contour plots of axial velocity for (a) the basic state, and (b) for the oscillatory secondary solution $(T=0.89)$ in four time instants obtained at $\mathrm{Ra}=7500$.

$\mathrm{Ra}_{c}=4.43 \times 10^{3}$, with the critical frequency $\omega_{c}=5.19$. This type of instability is dominant in the range of rotation rates $\Omega \in$ $(0,1.35)$. The secondary flow, thus, is a periodic orbit that does not preserve the $R$ symmetry, as can be observed in Fig. 12. This figure shows the velocity field at $z=0.5 \Gamma$ associated to the steady basic flow [Fig. 12(a)] and to the periodic flow in four time instants during a period ( $T=1.1$ in this case) for $\mathrm{Ra}=5500$ [Fig. 12(b)]. An inspection of the transverse (a)

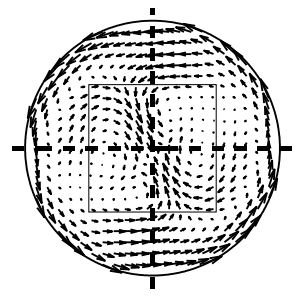

(c)

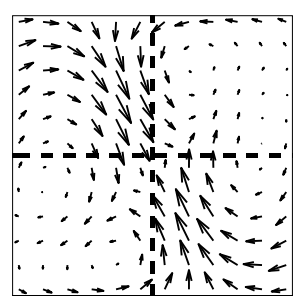

(b)

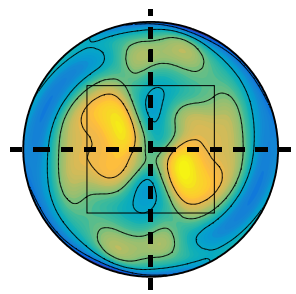

(d)

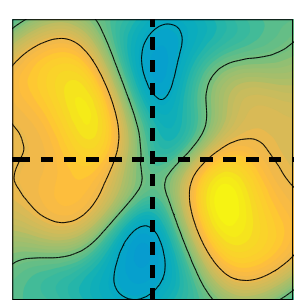

FIG. 14. Nonsymmetric steady flow for $\Omega=4.1$ arising from an instability in the main curve. The cross sections at $z=0.5 \Gamma$ correspond to (a) the transverse velocity field and (b) the contour plots of axial vorticity for the nonsymmetric steady solution obtained at $\mathrm{Ra}=6420$. [(c) and (d)] Enlargements of the central zone of the previous figures. velocity field (upper part of Fig. 12) reveals the presence of a vortex travelling along an elliptical trajectory inclined about $45^{\circ}$ with respect to a vertical diameter of the cross section. Also, when observing the evolution of the contour plots for the axial velocity (lower part of Fig. 12), we can appreciate that the zones with a higher magnitude of the axial velocity (light parts correspond to positive senses of axial velocity and dark parts to negative values) oscillate horizontally during the period; while at $T / 4$ both the maximum and minimum axial velocity zones are located at the left-hand side of the cell, they slowly shift and are located at the right-hand side at $3 T / 4$. Finally, as must happen in any Hopf bifurcation that breaks the reflection symmetry in a $Z_{2}$-symmetric system, the $R$ symmetry about the center of the cylinder is recovered after a time evolution of half a period.

In the range of rotation rates $\Omega \in(1.35,4.0)$, the character of the dominant instability changes, and the basic flow undergoes a symmetry-preserving oscillatory bifurcation. An example of the oscillatory flow triggered by this instability can be seen in Fig. 13(b), which shows, in a $z=0.5 \Gamma$ plane, the velocity field of a solution computed at $\mathrm{Ra}=7500$ when the rotation rate is $\Omega=2.3$. This Rayleigh number is above the critical value of the oscillatory bifurcation, which takes place at $\mathrm{Ra}_{c}=6.99 \times 10^{3}$ and has a critical frequency of $\omega_{c}=7.11$. Therefore, unlike in the previous case, this solution preserves the $R$ symmetry with respect to the center of the cell. Its appearance is similar to that of the basic flow [Fig. 13(a)], the only difference being the shape of the central vortex, which elongates and contracts periodically $(T=0.89)$, changing between an ellipse and a circle.

When the rotation rate is increased further, in the range $\Omega \in$ $(4.0,4.3)$, the dominant instability is a pitchfork bifurcation that breaks the $R$ symmetry. This critical curve of pitchfork bifurcations is represented with solid triangles in Fig. 9. 
The secondary solution shown in Fig. 14 for $\Omega=4.1$ and $\mathrm{Ra}=6420$ represents a stable steady solution obtained near the pitchfork bifurcation that takes place at a critical Rayleigh number $\operatorname{Ra}_{c}=6.29 \times 10^{3}$. We are plotting, at $z=0.5 \Gamma$, the transverse velocity field in Fig. 14(a) and to try to enhance the fluctuations with respect to the basic state, the axial vorticity rather than the axial velocity, in Fig. 14(b). The flow in the central part of the cylinder is enlarged in Fig. 14(c) and Fig. 14(d). A look at the figures reveals that the $R$ symmetry is broken. Axial vorticity in two symmetric points with respect to the center of the cell should be the same if the symmetry was kept, and this does not happen in Fig. 14(d). This difference can also be appreciated in Fig. 14(c), when comparing in this plane the length and orientation of the arrows crossing the positive and negative horizontal axis.

For values of rotation slightly above $\Omega=4.3$, the dominant instability is a low-frequency Hopf bifurcation that maintains the $R$ symmetry of the basic flow. This instability, represented with solid squares in Fig. 9, exhibits a steep decrease of the critical Rayleigh number with increasing rotation. Figure 15 shows the oscillatory flow obtained for $\Omega=4.4$ at $\mathrm{Ra}=$ 5810. This Rayleigh number is slightly above the critical Rayleigh number, since $\mathrm{Ra}_{c}=5.80 \times 10^{3}$ and $\omega_{c}=0.49$. The transverse velocity and the contour plots of axial vorticity at midplane show the presence of two vortices. Positive values of vorticity [light (yellow) parts], correspond to a clockwise rotating vortex, while negative values [dark (blue) parts], are associated to an counterclockwise rotating vortex. These vortices oscillate and change shape slightly within a period.

To complete the analysis of the nature of secondary flows that can arise in the region of the parameter space we are considering in this work, we inspect the features of two more stable oscillatory solutions obtained for $\Omega=3.8$ and $\Omega=4.5$. The first one is triggered by an instability in the disconnected curve of solutions, while the second one is obtained in the vicinity of an instability in the main curve of solutions but after its reconnection with the disconnected curve has taken place.

As already mentioned, the curve with open circles (blue) in Fig. 9 locates in the $(\Omega, \mathrm{Ra})$ diagram the position of the saddle nodes above which the basic flow coexists with the two additional steady $R$-invariant solutions (disconnected curve). The oscillatory flow presented in Fig. 16, obtained for $\Omega=3.8$ and $\mathrm{Ra}=5915$, is computed for a Rayleigh number above the symmetry-preserving oscillatory bifurcation indicated with open squares in Fig. 9, which takes place in a branch of the disconnected curve $\left(\mathrm{Ra}_{c}=5.79 \times 10^{3}\right.$, $\left.\omega_{c}=1.13\right)$. The critical curve locating these Hopf bifurcations of the stable branch of solutions extends from $\Omega=3.44$ to $\Omega=4.6$ and exhibits a discontinuity in $\Omega \approx 4.2$, as explained in the previous section. Again we are representing the axial vorticity instead of the axial velocity. Figure 16 shows the transverse velocity field and the contour plot of axial vorticity for both (a) the steady basic solution and (b) the periodic secondary solution $(T=8.8)$ in four time instants. Light (yellow) and dark (blue) values of axial vorticity correspond to a clockwise-counterclockwise rotating vortex. The contour plots of the axial vorticity show that the solution oscillates between the solutions of each one of the two branches of the disconnected curve. Notice that the contour plots of vorticity at $T / 4$ and 3T/4 in Fig 16 look similar to those of solutions belonging to the two branches of the disconnected curve and shown in the insets in Fig. 7. A detailed inspection of the dynamics of the bifurcated oscillatory solutions at this rotation rate, $\Omega=3.8$, shows that as the Rayleigh number slightly increases, the solution spends more and more time near the unstable steady solution of the branch from which it has not bifurcated, and that the period increases following a

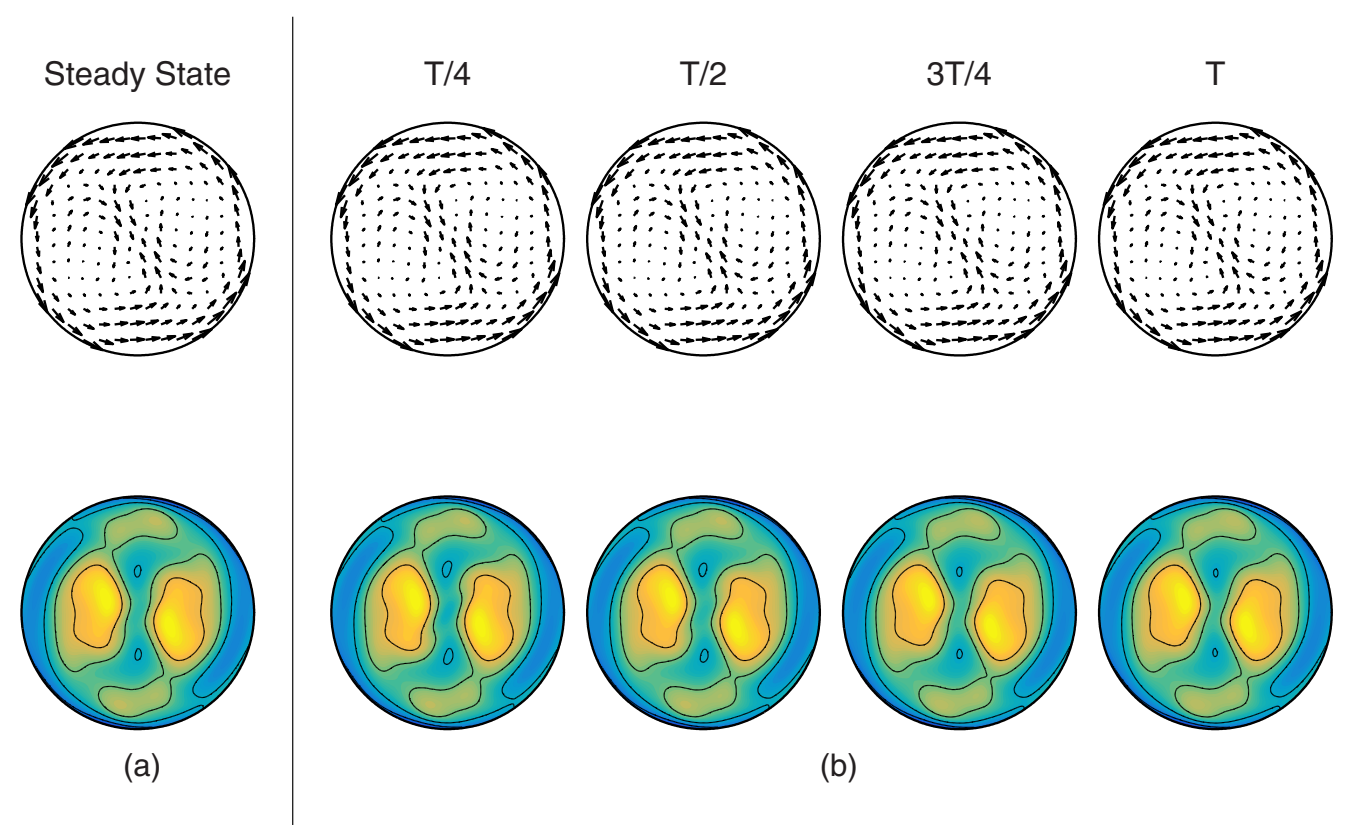

FIG. 15. Symmetric oscillatory flow for $\Omega=4.4$ arising from an instability in the main curve. The cross sections at $z=0.5 \Gamma$ correspond to the transverse velocity field and the contour plots of axial vorticity for (a) the basic state and (b) for the oscillatory secondary solution $(T=15.9)$ in four time instants obtained at $\mathrm{Ra}=5810$. 


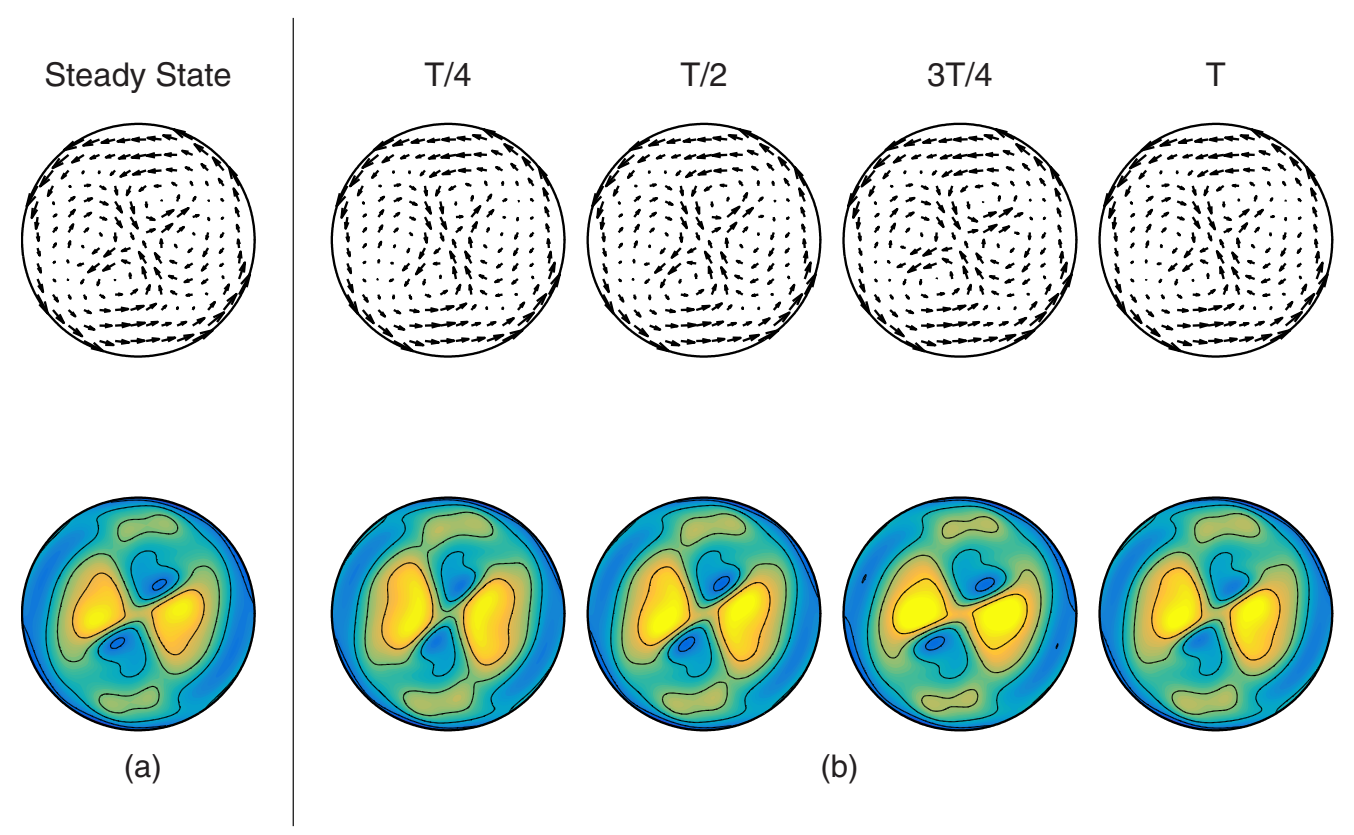

FIG. 16. Symmetric oscillatory flow for $\Omega=3.8$ arising from an instability in the disconnected curve. The cross sections at $z=0.5 \Gamma$ correspond to the transverse velocity field and contour plot of axial vorticity for (a) the basic state and (b) for the oscillatory secondary solution $(T=8.8)$ in four time instants obtained at $\mathrm{Ra}=5915$.

logarithmic divergence law. This behavior is consistent with an homoclinic connection (saddle-loop global bifurcation) near $\mathrm{Ra} \approx 5917$.

Finally, Fig. 17 shows the last secondary solution we have computed, which has been obtained for $\Omega=4.5$ at $\mathrm{Ra}=6250$ $\left(\mathrm{Ra}_{c}=6.12 \times 10^{3}, \omega_{c}=1.64\right)$. This state is triggered by the same symmetry-preserving oscillatory bifurcation, as in the case of the solution obtained for $\Omega=3.8$, but the location of the instability is indicated with solid squares in Fig. 9 rather than with open squares because, for this value of the rotation rate, the disconnected curve has already reconnected with the main curve; the instability, thus, takes place in the main curve. Whereas in the basic solution for $\Omega=3.8$ the intensity of the clockwise-counterclockwise central vortices was similar, we can appreciate in Fig. 17(a) that in this case the central counterclockwise-rotating vortices (dark) have damped and are less intense than the clockwise-rotating vortices (light). For this value of the Rayleigh number, unlike the behavior of the secondary solution that we have shown for $\Omega=3.8$, for this value of the Rayleigh number the secondary solution oscillates around the basic flow from which it has bifurcated. The spatiotemporal behavior shows that when the light areas shrink, the darker ones are reinforced and vice versa.

\section{SUMMARY AND CONCLUDING REMARKS}

In this paper, we have studied the flow in a horizontal closed cylinder of aspect ratio $\Gamma=2$ induced by both an axial temperature gradient and by rotation, for a Prandtl number $\sigma=0.01$. The main interest in this problem arises from crystal growth processes. The observation that oscillations in the solidification front can cause that crystals grown from the melt present inhomogeneities in the form of striations [1] motivated several studies with different geometrical configurations, which focused on the oscillatory threshold in low-Prandtlnumber fluids [2-4] characteristic of molten metals and semiconductors. In these works, the unique source of instability was the temperature gradient. Our present study incorporates rotation to the system analyzed in Ref. [12]. We have characterized the symmetric basic states and analyzed their stability when the temperature gradient increases for rotation rates in the range $\Omega \in(0,8)$, in which the two driving effects, natural convection and rotation, have been found to be comparable.

We have obtained numerically the fully three-dimensional basic flow by using a special preconditioned Newton's method [27], and we have used a continuation algorithm in the Rayleigh number and the rotation rate (parameter appearing only in the boundary condition) to follow the branches of solutions. The basic state, steady in the laboratory frame, consists of a longitudinal flow, symmetric with respect the center of the cylinder and tilted in the vertical direction. As the rotation increases, temperature becomes more uniform and the strength of the flow reduces due to buoyancy in the vertical direction (Fig. 5 and Fig. 6). These results are in agreement with those reported in previous works, where different values of the Prandtl number were used [13-15]. The continuation method used to follow steady solutions when rotation varies has allowed us to identify a novel result: The existence, for a fixed value of $\Omega$, of two curves of steady primary states. These two curves, a main and a disconnected one, have the same symmetric character and coexist for moderate values of the Rayleigh number, as occurrs in the nonrotating case for values of the Prandtl number around $\sigma \approx 0.025$ [12]. The presence of these two curves could be responsible for the anomalous variations of the dimensionless axial velocity with the Strouhal number that was observed for high values of the Grashof number in the work of Ref. [14]. 


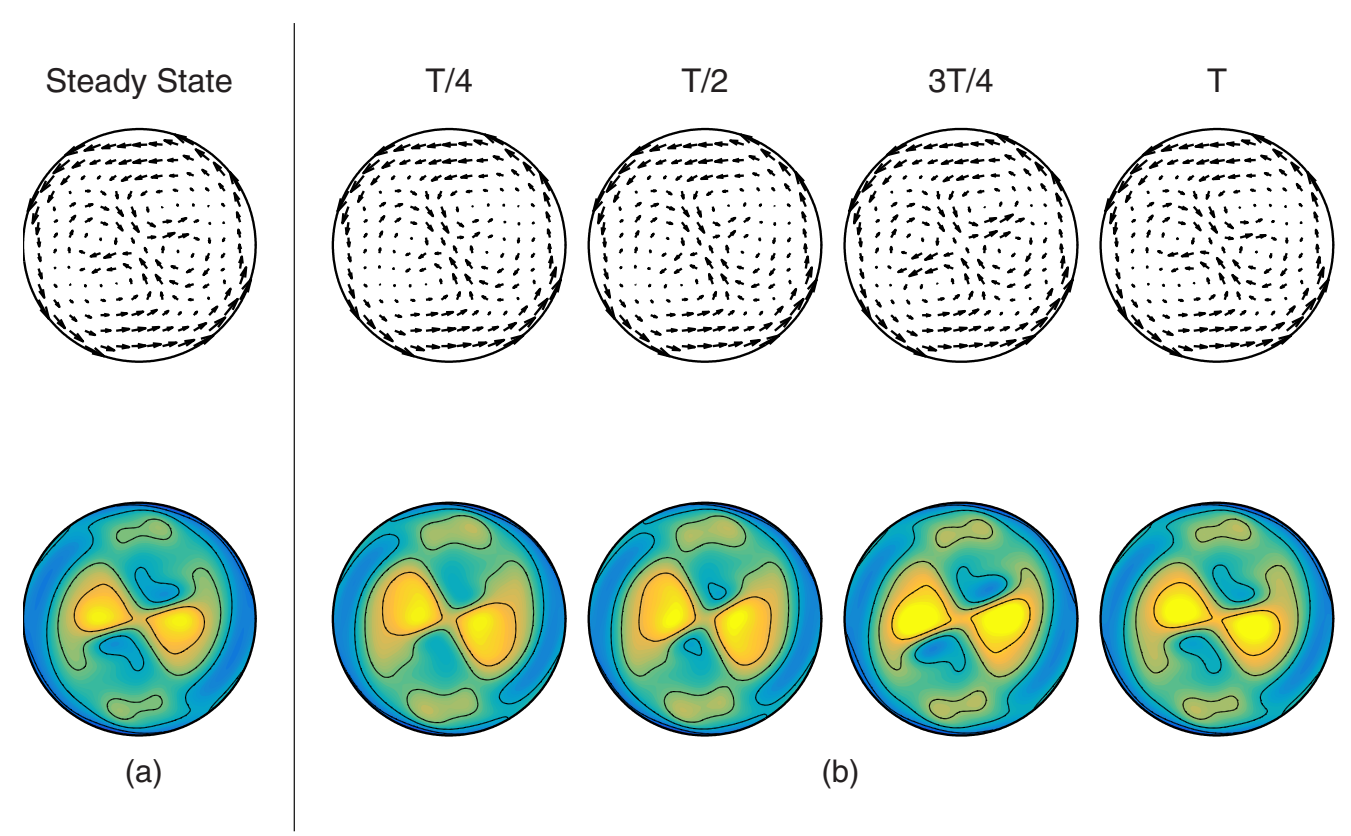

FIG. 17. Symmetric oscillatory flow for $\Omega=4.5$ arising from an instability in the main curve. The cross sections at $z=0.5 \Gamma$ correspond to the transverse velocity field and contour plot of axial vorticity for (a) the basic state and (b) for the oscillatory secondary solution ( $T=4.30)$ in four time instants obtained at $\mathrm{Ra}=6250$.

After the obtention of the basic states, we have analyzed their stability by calculating the leading eigenvalues along the branches of primary solutions to determine the instability threshold. The main results are summarized in Fig. 8 and Fig. 9. A first outstanding result concerns the role of rotation: Contrary to what might be expected, only for very small rotation rates, $\Omega \in(0,1.33)$, does rotation have a stabilizing effect. In this range of $\Omega$, the bifurcation responsible for this instability is oscillatory and breaks the symmetry of the basic state. For $\Omega \in(1.33,4)$, primary solutions in the main curve lose stability in a Hopf bifurcation that maintains the symmetry and whose value of the critical Rayleigh and Hopf frequencies hardly vary with $\Omega$. When the value of the rotation rate approaches $\Omega=3.5$ and $\Omega=4.5$, the instabilities of the basic flow take place for lower values of the Rayleigh number, and complex scenarios of bifurcations related with the presence of very close bifurcations of codimension 2 arise. For $\Omega=3.5$ (Fig. 10), the scenario of bifurcations involves only the disconnected curve and sets the value of $\Omega$ from which the solutions belonging to one of the branches of the disconnected curve are stable, giving rise to a region in the $(\Omega, \mathrm{Ra})$ parameter space where two different stable steady symmetric solutions coexist. For $\Omega=4.5$ (Fig. 11), the scenario of bifurcations involves both curves of symmetric solutions and sets the point from which only one steady symmetric solution is stable. This happens after the cusp bifurcation near $\Omega \approx 4.6$.

Once the nature of the instabilities of the primary solutions is identified and the bifurcations are located in the parameter space, we have performed an energy analysis of the dominant eigenfunctions for the leading instabilities. This analysis has allowed us to elucidate whether the dominant instability mechanism is buoyancy or shear driven. We conclude that the instability is mainly dominated by shear forces, although the significance of the leading effect decreases as the rotation rate increases.

Finally, by using a time evolution code to integrate the governing equations, we have obtained the secondary flows arising from the most representative instabilities in the region of the parameter space we are considering. We have characterized the structure of the flows to gain some insight in the physical mechanisms leading to them. It is worth mentioning that in one of the explorations we have identified time-dependent secondary solutions oscillating between two solutions belonging to each one of the two branches of the disconnected curve. The period of the solutions follows a logarithmic divergence law consistent with an homoclinic connection to an unstable steady solution (saddle-loop global bifurcation). Remarkably, this homoclinic connection is apparently not related to any of the unfoldings of the two Takens-Bogdanov bifurcations we have identified.

We expect that the complex bifurcation scenarios we have identified for moderate values of the Rayleigh number and slow rotation rates lead to complex spatiotemporal dynamics, as it occurred in other analogous two-dimensional laterally heated systems, with similar aspect ratios and comparable values of the Prandtl number, that we had analyzed in the past [8-11]. However, a complete study of this emerging nonlinear dynamics would merit further study and is beyond the scope of this paper.

\section{ACKNOWLEDGMENTS}

The authors thank Dr. Esteban Meca for useful discussions and comments. This work is funded by DGICYT under Grant No. FIS2013-40880-P. O.S. was supported by FI-DGR2012 grant from the DGR of the Generalitat de Catalunya. 
[1] D. T. J. Hurle, E. Jackeman, and C. P. Johnson, J. Fluid. Mech. 64, 565 (1974).

[2] A. Y. Gelfgat, P. Z. Bar-Yoseph, and A. L. Yarin, J. Fluids Eng. 119, 823 (1997).

[3] S. Vaux, H. Ben Hadid, and D. Henry, J. Cryst. Growth 290, 674 (2006).

[4] D. Henry and H. Ben Hadid, Phys. Rev. E 76, 016314 (2007).

[5] M. Lappa, C. R. Mecanique 335, 261 (2007).

[6] T. P. Lyubimova, D. V. Lyubimov, V. A. Morozov, R. V. Scuridin, H. Ben Hadid, and D. Henry, J. Fluid Mech. 635, 275 (2009).

[7] D. V. Lyubimov, A. V. Burnysheva, H. Ben Hadid, T. P. Lyubimova, and D. Henry, J. Fluid Mech. 664, 108 (2010).

[8] I. Mercader, O. Batiste, and X. Ruiz, Theor. Comput. Fluid Dyn. 18, 221 (2004).

[9] E. Meca, I. Mercader, O. Batiste, and L. Ramírez-Piscina, Phys. Rev. Lett. 92, 234501 (2004).

[10] I. Mercader, O. Batiste, L. Ramírez-Piscina, X. Ruiz, S. Rüdiger, and J. Casademunt, Phys. Fluids 17, 104108 (2005).

[11] E. Meca, I. Mercader, and L. Ramírez de la Piscina, Physica D 303, 39 (2015).

[12] I. Mercader, O. Sánchez, and O. Batiste, Phys. Fluids 26, 014104 (2014).

[13] H. Q. Yang, K. T. Yang, and J. R. Lloyd, AIChE J. 34, 1627 (1988).

[14] S. Fujiwara, Y. Watanabe, Y. Namikawa, T. Keishi, K. Matsumoto, and T. Kotani, J. Cryst. Growth 192, 328 (1998).

[15] C. W. Lan and C. Y. Tu, J. Cryst. Growth 237-239, 1881 (2002).

[16] F. Ladeinde and K. E. Torrance, J. Fluid. Mech. 228, 361 (1991).
[17] M. Zhao, L. Robillard, and P. Vasseur, Int. Comm. Heat Mass Transfer 25, 1031 (1998).

[18] I. Mercader, O. Batiste, and A. Alonso, Comput. Fluids 39, 215 (2010).

[19] S. Hugues and A. Randriamampianina, Int. J. Numer. Meth. Fluids 28, 501 (1998).

[20] B. Fornberg, A Practical Guide to Pseudospectral Methods (Cambridge University Press, Cambridge, 1998).

[21] L. N. Trefethen, Spectral Methods in Matlab (SIAM, Philadelphia, 2000).

[22] I. Mercader, M. Net, and A. Falqués, Comp. Meth. Appl. Mech. Eng. 91, 1245 (1991).

[23] I. Mercader, A. Alonso, and O. Batiste, Phys. Rev. E 77, 036313 (2008).

[24] A. Alonso, I. Mercader, and O. Batiste, Fluid Dyn. Res. 46, 041418 (2014).

[25] F. Marques, I. Mercader, O. Batiste, and J. Lopez, J. Fluid Mech. 580, 303 (2007).

[26] J. Lopez, F. Marques, I. Mercader, and O. Batiste, J. Fluid Mech. 590, 187 (2007).

[27] C. K. Mamun and L. S. Tuckerman, Phys. Fluids 7, 80 (1995).

[28] V. Frayssé, L. Giraud, S. Gratton, and J. Langou, Technical Report TR/PA/03/3, CERFACS; Public domain software available on www.cerfacs/algor/Softs (2003).

[29] E. Chenier, C. Delcarte, and G. Labrosse, Phys. Fluids 11, 527 (1999).

[30] S. H. Xin and P. Le Quéré, Phys. Fluids 13, 2529 (2001).

[31] D. Ma, D. Henry, and H. Ben Hadid, Phys. Fluids 17, 124101 (2005). 